ARTICLE

\title{
A cluster of noncoding RNAs activates the ESR1 locus during breast cancer adaptation
}

\author{
Saori Tomita ${ }^{1,2, \star}$, Mohamed Osama Ali Abdalla1,*, Saori Fujiwara ${ }^{1,2}$, Haruka Matsumori ${ }^{1}$, Kazumitsu Maehara ${ }^{3}$,
} Yasuyuki Ohkawa ${ }^{3}$, Hirotaka Iwase ${ }^{2}$, Noriko Saitoh ${ }^{1} \&$ Mitsuyoshi Nakao 1,4,5

Estrogen receptor- $\alpha$ (ER)-positive breast cancer cells undergo hormone-independent proliferation after deprivation of oestrogen, leading to endocrine therapy resistance. Up-regulation of the ER gene (ESR1) is critical for this process, but the underlying mechanisms remain unclear. Here we show that the combination of transcriptome and fluorescence in situ hybridization analyses revealed that oestrogen deprivation induced a cluster of noncoding RNAs that defined a large chromatin domain containing the ESR1 locus. We termed these RNAs as Eleanors (ESR1 locus enhancing and activating noncoding RNAs). Eleanors were present in ER-positive breast cancer tissues and localized at the transcriptionally active ESR1 locus to form RNA foci. Depletion of one Eleanor, upstream (u)-Eleanor, impaired cell growth and transcription of intragenic Eleanors and ESR1 mRNA, indicating that Eleanors cis-activate the ESR1 gene. Eleanor-mediated gene activation represents a new type of locus control mechanism and plays an essential role in the adaptation of breast cancer cells.

\footnotetext{
${ }^{1}$ Department of Medical Cell Biology, Institute of Molecular Embryology and Genetics, Kumamoto University, Kumamoto 860-0811, Japan. ${ }^{2}$ Department of Breast and Endocrine Surgery, Graduate School of Medical Sciences, Kumamoto University, Kumamoto 860-8556, Japan. ${ }^{3}$ Department of Advanced Initiative Medicine, Faculty of Medicine, Kyushu University, Fukuoka 812-8582, Japan. ${ }^{4}$ Program for Leading Graduate Schools 'HIGO (Health life science: Interdisciplinary and Glocal Oriented) Program', Kumamoto University, Kumamoto 860-8556, Japan. ${ }^{5}$ Core Research for Evolutional Science and Technology (CREST), Japan Science and Technology Agency, Tokyo 102-0076, Japan. * These authors contributed equally to this work. Correspondence and requests for materials should be addressed to N.S. (email: norikos@kumamoto-u.ac.jp) or to M.N. (email: mnakao@gpo.kumamoto-u.ac.jp).
} 
C ancer cells adapt to the surrounding environment and maintain their proliferation, resulting in malignant transformation and resistance to anticancer treatments ${ }^{1}$. Breast cancers expressing estrogen receptor- $\alpha$ (ER) depend on oestrogen for cellular growth and survival. ER functions as a nuclear receptor-type transcription factor upon binding to oestrogen and regulates the expression of various target genes. Endocrine therapies, such as the use of an aromatase inhibitor (AI) that blocks oestrogen production, are the most effective for ER-positive breast cancers ${ }^{2}$. However, these treatments are frequently followed by disease recurrence because most breast tumours, which are initially responsive to these therapies, develop resistances through unknown mechanisms ${ }^{2-4}$.

MCF7 human breast cancer cells are ER-positive and acquire oestrogen-independent proliferation when they are cultured under an oestrogen-depleted condition for a prolonged period of time (long-term oestrogen deprivation; LTED) $)^{5,6}$. LTED adaptation is a well-established cellular model that recapitulates acquisition of AI resistance or postmenopausal tumorigenesis ${ }^{5-10}$. Previous studies have reported that the gene-encoding ER (ESR1) is up-regulated during LTED adaptation ${ }^{8}$, which is found in ERpositive human breast cancers. Understanding the molecular mechanism of this gene activation is critical because overproduction of ER may lead to an enhanced response to low concentrations of oestrogen, which is responsible for the LTEDadapted phenotype e $^{7,9,11}$. Paradoxically, administration of oestrogen is an effective treatment for AI-resistant breast cancers $^{12-14}$, and the LTED cell model may be used to gain the mechanistic evidence for such therapeutic efficacy.

Gene expression patterns are reprogrammed in response to environmental changes or during development and linked to the conversion of cellular phenotypes. Several events that occur in chromatin include recruitment of transcriptional activators/ repressors, changes in histone/DNA modifications, RNA polymerase II (RNA Pol II) binding, long-range chromosomal interactions and chromatin domain formation ${ }^{15-18}$. It was classically shown that the $\beta$-globin locus forms a distinct open chromatin domain during erythropoiesis ${ }^{16-18}$. To date, various types of chromatin domains have been characterized as $\sim 10 \mathrm{~kb}$ to a few $\mathrm{Mb}$ in length by genome-wide chromosome conformation, histone modification patterns, association with specific nuclear architectures and nuclease sensitivities ${ }^{19-22}$. These data suggest that interphase chromosomes are organized by hierarchical folding through which transcription can be regulated through chromatin domain formation.

Recent studies have revealed that noncoding RNAs (ncRNAs) are also involved in transcriptional regulation through diverse functions ${ }^{23}$. The mammalian transcriptome includes thousands of long noncoding RNAs (lncRNAs) that are longer than 200 nucleotides and devoid of protein-coding potential ${ }^{24}$. Some lncRNAs show unique expression under specific conditions such as $\mathrm{X}$ chromosome inactivation, genomic imprinting and maintenance or differentiation of stem cells ${ }^{25-27}$. LncRNAs are encoded at virtually any site of the genome, including enhancer, promoter, intron and intergenic regions, which regulate genes both in cis and trans. Currently, the potential roles of lncRNAs in cancer cell adaptation are unknown.

In the present study, we found that up-regulation of ESR1 was important for LTED cell adaptation, which was maintained by novel ncRNAs produced from a large chromatin domain of the ESR1 gene. Fluorescence in situ hybridization (FISH) analyses showed that these ncRNAs, termed Eleanors (ESR1 locus enhancing and activating noncoding RNAs), were localized at the site of active transcription, resulting in the formation of distinct RNA foci in the nucleus. One of the Eleanors, upstreamEleanor (u-Eleanor), originated from an enhancer-like sequence upstream of the ESR1 gene, which was necessary for enhanced expression of both ESR1 mRNA and intragenic Eleanors in LTED cells. Our genome-wide transcriptome analyses revealed that coordinated expression of ncRNA and mRNA, exemplified by the ESR1 gene, was conserved in a set of long genes. These findings uncover the molecular basis for endocrine therapyresistant breast cancer, which involves a new type of ncRNAmediated regulation of a chromatin domain and protein-coding genes.

\section{Results}

ESR1 up-regulation is accompanied by Eleanor expression. To understand the mechanism of hormonal adaptation and the action of resveratrol in ER-positive breast cancers, we used a cell model system in which MCF7 cells were cultured under three different conditions: normal (MCF7), oestrogen deprivation for 2-4 months (LTED) and further treatment with $100 \mu \mathrm{M}$ resveratrol for $24 \mathrm{~h}$ (LTED-RES, Fig. 1a). Resveratrol is structurally similar to oestrogen, binds to ER in vitro and exerts oestrogenic effects on breast cancer cells ${ }^{28,29}$. Quantitative PCR with reverse transcription (qRT-PCR) and immunofluorescence analyses showed that ESR1 expression was significantly increased in LTED cells and dramatically suppressed by resveratrol (Fig. 1b,c). Notably, knockdown of ER significantly reduced LTED cell proliferation at $96 \mathrm{~h}$ after transfection of the small interfering RNA (siRNA) (Fig. 1d). This result suggests that the up-regulation of ER plays a role in acquisition of oestrogenindependent cancer cell growth.

To further investigate activation of the ESR1 gene, we performed mRNA-Seq and RNA-Seq analyses of cells under the three conditions. We prepared poly (A) ${ }^{+}$RNA for mRNA-Seq, and total RNA that was devoid of ribosomal RNA for RNA-Seq, respectively (see Methods for details). Gene tracks representing mRNA-Seq and RNA-Seq data are shown in Fig. 1e. The human ESR1 locus resides on chromosome 6, consists of eight exons and is $\sim 300 \mathrm{~kb}$ in length. As expected, mRNA-Seq data showed up-regulation of ESR1 exons in LTED cells and repression in LTED-RES cells (Fig. 1e, top three tracks). Interestingly, RNA-Seq analyses detected a significant amount of intragenic transcripts in LTED cells, which extended along the entire ESR1 locus including introns and upstream noncoding regions, but not to the neighbouring silent gene, SYNE1 (Fig. 1e, fifth track). We named the noncoding RNAs produced from inside and around the ESR1 locus (6q25.1; 152083078-152424447) as 'Eleanors'. Production of Eleanors was well synchronized with production of ESR1 mRNA, suggesting that Eleanors participate in regulation of the ESR1 gene. Eleanors were distinct from previously reported types of ncRNAs, such as enhancer/promoter-RNAs and miRNAs, because Eleanors were expressed from a much larger chromatin region.

Eleanors are localized at the site of ESR1 transcription. To confirm the presence of Eleanors, we assessed transcripts from ESR1-intron 2 by qRT-PCR (Fig. 2a). Generally, introns are rapidly processed to undetectable levels after transcription as shown for intron 11 of ERBB2. However, transcripts from intron 2 of ESR1 were unusually stable in LTED cells. To further examine the presence of ncRNAs derived from the broad region of the ESR1 locus, we performed FISH analyses using bacterial artificial chromosome (BAC) probes that covered most of the ESR1 locus (ESR1-BAC) (Figs 1e and 2b). First, cells were processed for DNA FISH using the BAC clone for the centromeric region of chromosome 6 (CEN6-BAC) as a control (Fig. 2b, top panels). We detected three or four CEN6 signals (red) in the nucleus because of the standard karyotypes of MCF7 cells ${ }^{30}$. As expected, ESR1 signals (green) were detected close to each CEN6 
a

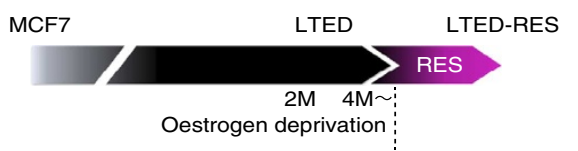

C

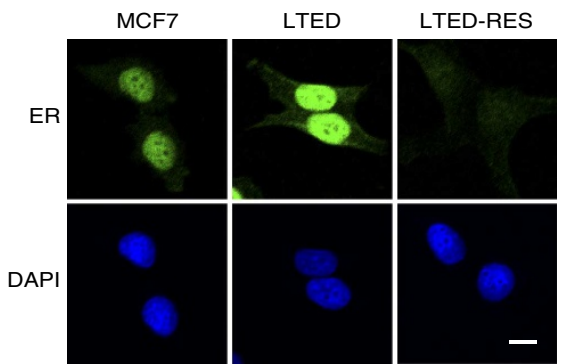

b

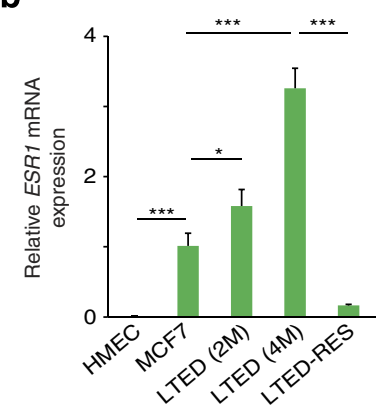

d

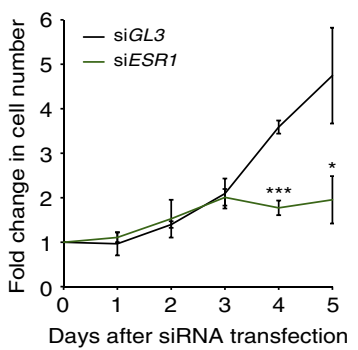

e

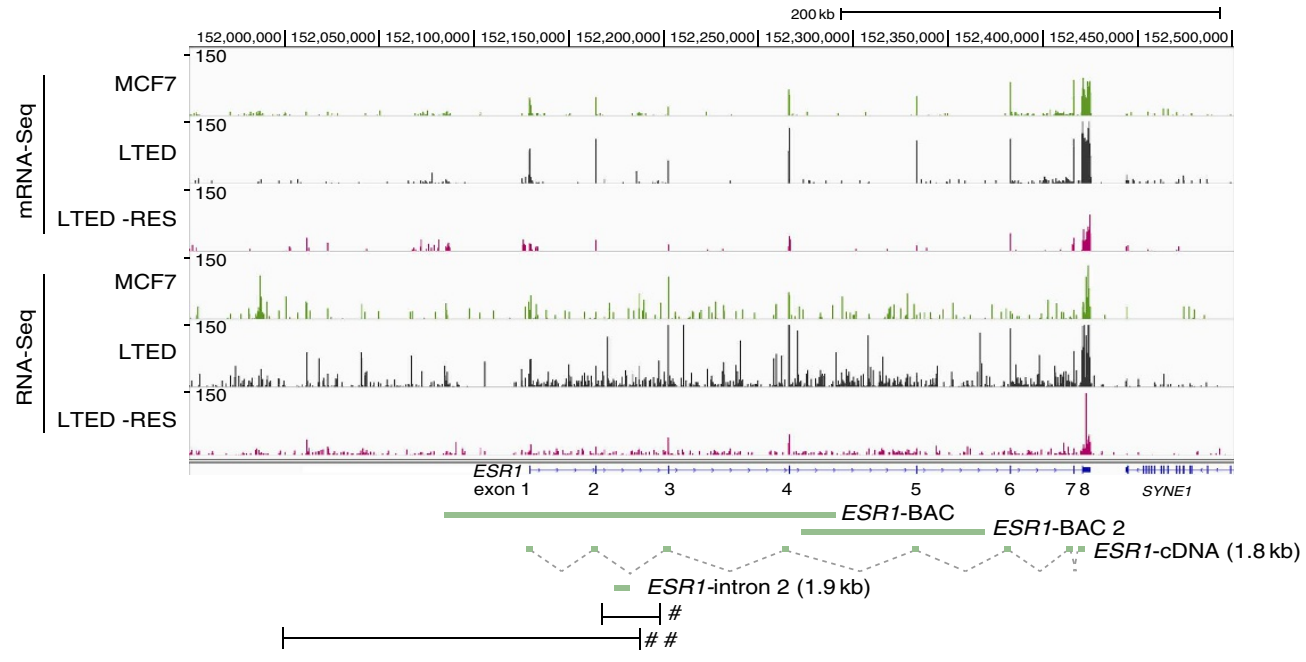

Figure 1 | Eleanors and ESR1 mRNA are coordinately expressed in LTED and LTED-RES cells. (a) Schematic representation of the cell models used in this study. ER-positive MCF7 breast cancer cells were cultured under three conditions: MCF7, LTED and LTED-RES. (b) Expression levels of ESR1 mRNA. qRT-PCR results under the MCF7 condition were set to 1. Primers were designed to cover the exon-exon junction. Values are the means \pm s.d.; $n=3$. $P$-values were calculated using Student's $t$-test $\left({ }^{\star} P<0.05,{ }^{\star \star \star} P<0.001\right)$. (c) Immunofluorescence of ER showing enhanced expression in LTED cells and its suppression by resveratrol treatment (LTED-RES). Scale bar, $10 \mu \mathrm{m}$. (d) ESR1 knockdown inhibits LTED cell proliferation. LTED cells were treated with siRNA targeting ESR1 for the indicated periods. Cell growth is shown as fold changes. Values are the means \pm s.d.; $n=3$. $P$-values were calculated using Student's $t$ test $\left({ }^{\star} P<0.05,{ }^{\star \star} P<0.001\right)$. (e) Gene tracks representing mRNA-Seq and RNA-Seq data of the human ESR1 locus. Novel ncRNAs, termed Eleanors, were abundantly expressed in LTED cells from the entire ESR1 locus, which were detected as read signals in non-exonic regions. Eleanors were suppressed in LTED-RES cells. The structures of ESR1 and downstream SYNE1 genes are shown below. Green bars indicate the FISH probes used in this study. Regions highlighted in Figs $2 \mathrm{a}$ and $4 \mathrm{a}$ are denoted by \# and \#\#, respectively. 2M, two months; $4 \mathrm{M}$, four months.

signal. However, it was surprising that the ESR1 signals were significantly larger in LTED cells ( 2-fold higher ESR1/CEN6 area ratio compared with that in MCF7 cells) and obviously smaller in LTED-RES cells (Fig. 2b, bottom right panel).

The enlarged FISH signals may suggest homogeneously staining regions that are cytogenetic hallmarks of genomic amplification in cancer ${ }^{31}$. However, such a notion was not the case for the ESR1 locus in LTED cells, because the enlarged foci were promptly reduced to small dots by resveratrol treatment. Indeed, we found no ESR1 gene amplification in copy number variation analysis (Supplementary Fig. 1a,b).
Further analyses showed that most of the enlarged FISH signals consisted of RNA molecules, because they were significantly diminished by treatment with RNase, but not DNase (Supplementary Fig. 1c). To clarify the origin of the RNAs accumulating in the enlarged foci, we performed FISH under non-denaturing conditions (RNA FISH) using the ESR1-BAC probe (covering noncoding regions as well as exons), ESR1-cDNA probe (covering exons exclusively) and ESR1-intron 2 probe (Fig. 2b lower three panels). The enlarged signals in LTED cells were detected with the BAC and intron 2 probes, but not the ESR1-cDNA probe, indicating that ncRNAs derived from the 
intragenic region (Eleanors) were the major components of the enlarged FISH signals.

We investigated the spatial positioning of Eleanors relative to the ESR1 locus in the LTED nucleus by sequential hybridizations. First, Eleanors were hybridized with the ESR1-BAC2 probe (shown in Fig. 1e), followed by fixation of the signals and subsequent RNase treatment, and then the ESR1 gene was hybridized with the ESR1-BAC probe. The results showed that Eleanors and the ESR1 locus were co-localized in the nucleus (Fig. 2c). Collectively, these data demonstrate that Eleanors are produced from the intragenic region of the transcriptionally active ESR1 locus in LTED cells and remain associated with the site of transcription, resulting in the formation of distinct RNA foci. Our stranded RNA-Seq results indicated that a

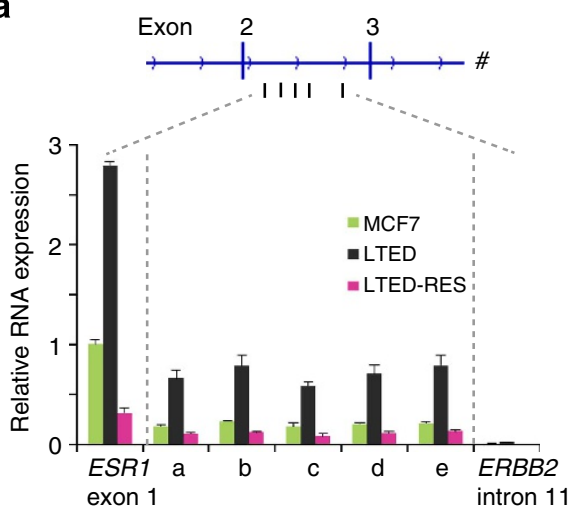

C

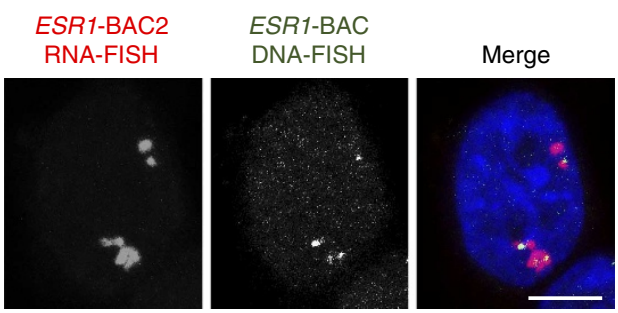

b

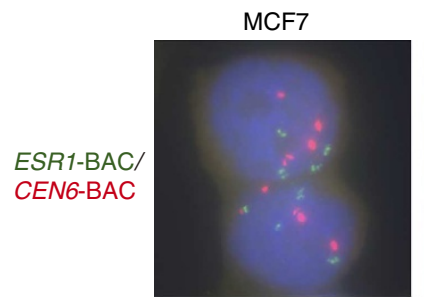

ESR1-BAC

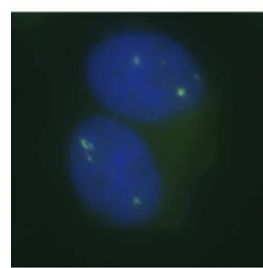

ESR1-CDNA
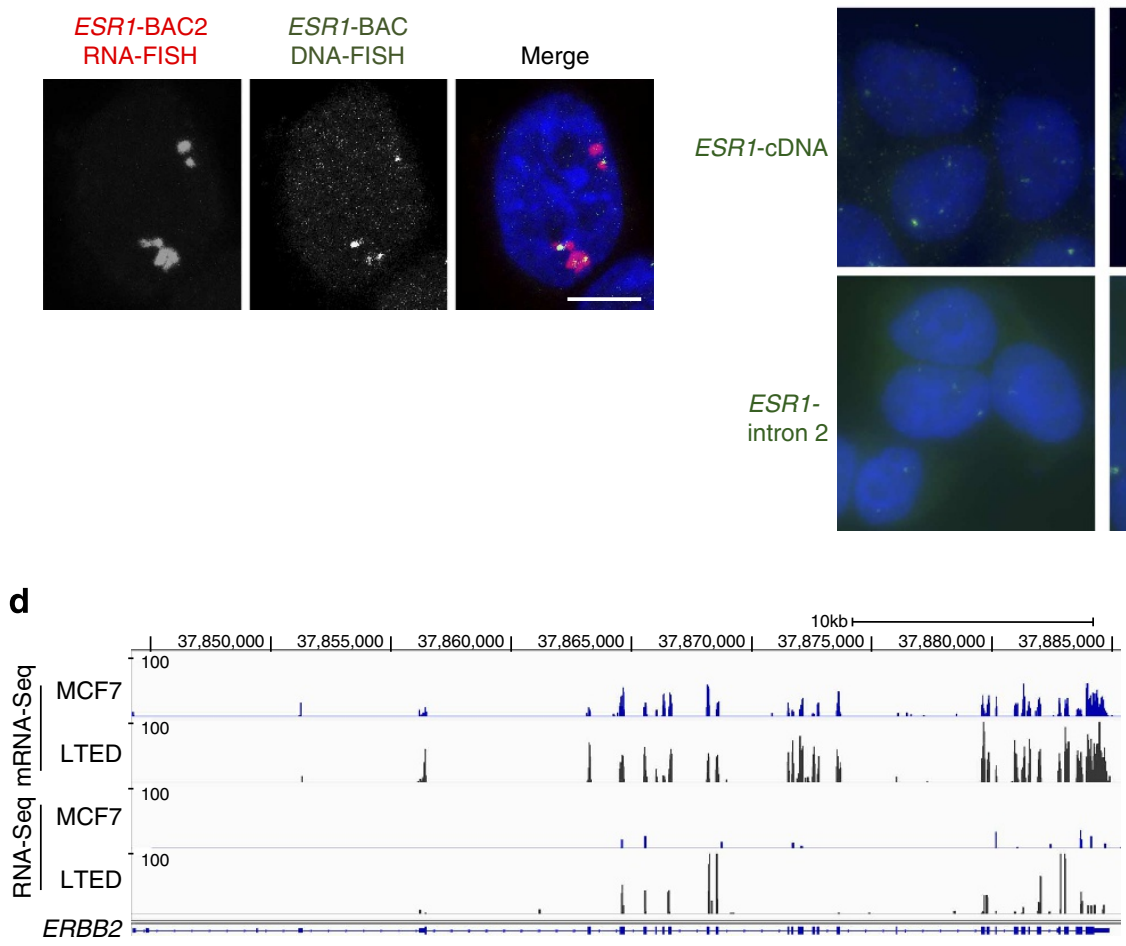

LTED
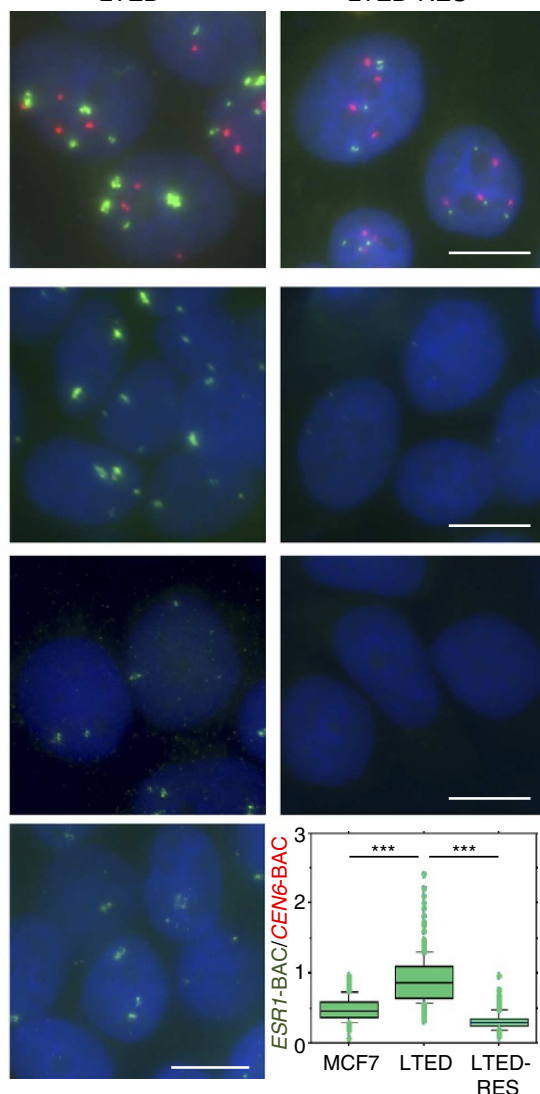

LTED-RES
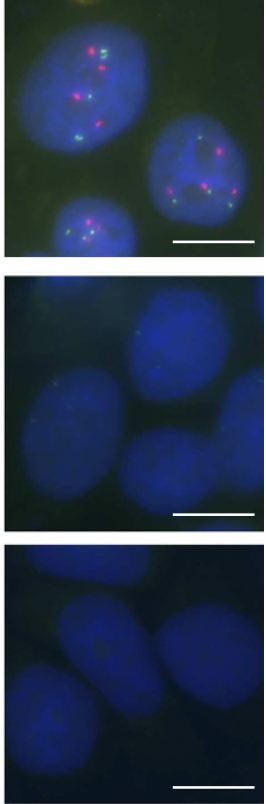

e

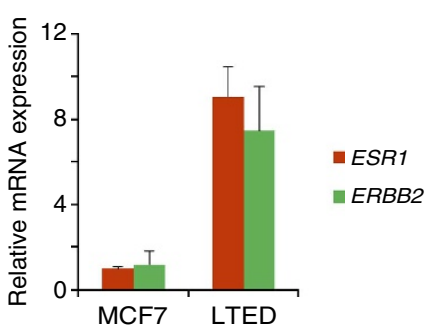

$\mathbf{f}$

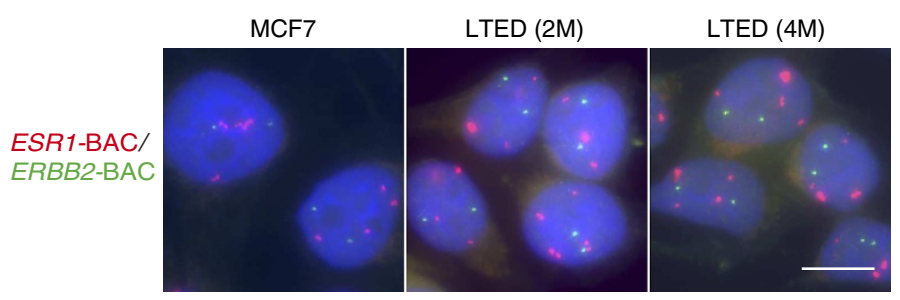

g

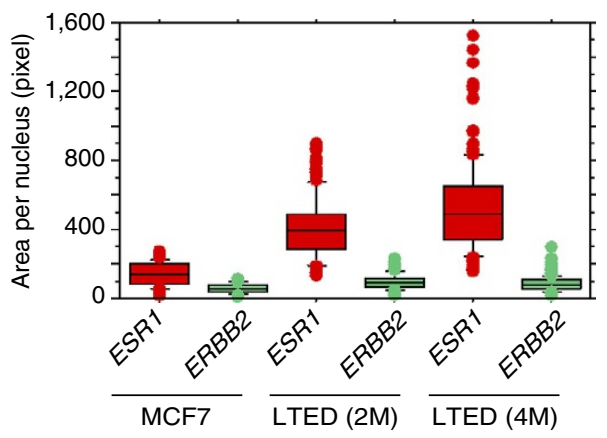


Eleanors were transcribed in the same direction as that of ESR1 mRNA.

To investigate whether ncRNA production in LTED cells is specific to the ESR1 locus, we examined the ERBB2 gene that plays a role in a subset of breast cancers. Gene tracks representing mRNA-Seq and RNA-Seq data of the ERBB2 locus are shown in Fig. 2d. ERBB2 gene expression was not accompanied by ncRNA production in LTED cells. However, similar to ESR1 expression, $E R B B 2$ expression was activated by more than 3-fold (Fig. 2e). Consistently, FISH signals for the ERBB2 locus (green) showed no changes, while signals for the ESR1 locus (red) were large in the same nucleus (Fig. 2f,g). Two other genes, APP and ERGIC, also showed similar levels of up-regulation in LTED cells without significant RNA expression from noncoding regions (Supplementary Fig. 2).

Adaptation to hormone deprivation is conserved among several breast cancer cells. Another ER-positive breast cancer cell line, HCC1428 acquires oestrogen-independent proliferation with up-regulation of ER after a long period of oestrogen deprivation (HCC1428 LTED cells) ${ }^{32}$. Using an ESR1-BAC probe, we detected the enlarged FISH signals in HCC1428 LTED cells, which were suppressed by resveratrol treatment (HCC1428 LTED-RES cells), similar to LTED and LTED-RES cells (Supplementary Fig. 1d).

Together, the Eleanor-containing foci were characteristic of the ESR1 locus in LTED cells, suggesting a new type of gene activation mechanism for the specific gene locus.

Eleanors are present in ER-positive breast cancer tissues. Naturally occurring human breast cancers are grouped into at least three subtypes: a luminal type that is ER-positive, an ERBB2 type that highly expresses ERBB2 with genomic amplifications, and a triple-negative type that is negative for ER, ERBB2 and the progesterone receptor ${ }^{33,34}$. To confirm the appearance of Eleanor-containing foci in vivo, we performed a combination of immunohistochemistry (IHC) and FISH analyses using serial sections of various breast cancer tissues (Fig. 3). We detected the enlarged FISH signals for Eleanors (green) in some luminal-type breast cancers, which were well correlated to ER overexpression found in IHC. On the other hand, these FISH signals were absent from normal breast tissue as well as ERBB2-type and triplenegative-type cancer tissues, all of which were ER negative. These results suggest significant implications of Eleanors in ER-positive breast cancer cells.

u-Eleanor enhances intragenic Eleanor and ESR1 mRNA. Our RNA-Seq data showed that the region producing ncRNAs extended further upstream of the ESR1 gene (Fig. 4a). One of the peaks on the gene track was positioned at site $\mathrm{c}$ that was $\sim 40 \mathrm{~kb}$ upstream of the canonical promoter A (site f) in MCF7 cells ${ }^{35,36}$. qRT-PCR analyses showed local transcription from site $c$ (Fig. 4b). Stranded RNA-Seq data indicated that $u$-Eleanor was transcribed in the same direction as that of intragenic Eleanors and ESR1 mRNA. In agarose gel electrophoresis of RT-PCR products, we detected a transcript of at least 1,200 nucleotides in length (Supplementary Fig. 3a,b). We termed this lncRNA as $u$-Eleanor.

Alignment with the chromatin immunoprecipitationsequencing (ChIP-Seq) data revealed that the $u$-Eleanor gene region (site c) was bound by RNA Pol II, CTCF and transactivators (GATA3, CEBPB and p300) in MCF7 cells (Supplementary Table 1). Consistently, this site was enriched with active histone marks (trimethyl-H3K4 and acetyl-H3K27) and devoid of repressive marks (trimethyl-H3K9 and trimethyl$\mathrm{H} 3 \mathrm{~K} 27)$. These active chromatin features at the $u$-Eleanor gene region were characteristic of cell types of breast origin. Similar to enhancer RNAs (eRNAs), these data suggest that site $c$ functions as an upstream regulatory element that is actively transcribed into ncRNAs ${ }^{37}$

Alternatively, $u$-Eleanor could be an unannotated upstream promoter of ESR1. Although promoter A is most frequently used in MCF7 cells ${ }^{36}$, transcription of ESR1 can be complex under certain circumstances because of the nature of differential promoter usage ${ }^{35,36}$. Gene annotations in UCSC and GENCODE genome browsers ${ }^{38,39}$ revealed a variety of cDNAs and ESTs, including independent transcripts, which terminate before the ESR1 gene, as well as a transcript that spans from the upstream region to the complete body of ESR1 (Supplementary Fig. 3a). However, $u$-Eleanor did not correspond to any of the previously described alternative promoters ${ }^{35,36}$. $u$-Eleanor was unlikely to be contiguous with the downstream Eleanors because we did not detect any transcripts from sites $\mathrm{d}$ or e (Fig. $4 \mathrm{~b}$ and Supplementary Fig. 3a,b). In addition, RT-PCR using several primer sets starting in $u$-Eleanor and ending in ESR1 exon 1 failed to detect any transcripts (cg in Supplementary Fig. 3b). Furthermore, no protein-coding possibility was predicted in the $u$-Eleanor gene region (Supplementary Table 5). Taken together, we conclude that $u$-Eleanor is a transcript independent of intragenic Eleanors and ESR1 mRNA.

Transcription of $u$-Eleanor may enhance ESR1 mRNA expression, because both RNAs were up-regulated in LTED cells and repressed in LTED-RES cells (Figs $1 \mathrm{~b}$ and $4 \mathrm{~b}$ ). We suspected that $u$-Eleanor might function as an eRNA, which was in agreement with our ChIP-qPCR data showing that the $u$-Eleanor region and promoter $A$ of $E S R 1$ were bound by the active form of RNA Pol II (phosphorylated at serine 5) in LTED cells (Fig. 4c). In addition, $u$-Eleanor chromatin was enriched with monomethylation of $\mathrm{H} 3 \mathrm{~K} 4$ (H3K4me1) rather than tri-methylation (H3K4me3) (Supplementary Fig. 3c,d), suggesting that this region

Figure 2 | Eleanors are associated with the site of ESR1 transcription in the nucleus. (a) Expression of Eleanor transcripts from various sites (a-e) within intron 2 of the ESR1 gene. ESR1 exon 1 and ERBB2 intron 11 were used as controls. For qRT-PCR, total RNA was pre-treated with DNase I. The value for ESR1 exon 1 in MCF7 cells was set to 1. Values are the means \pm s.d.; $n=3$. Corresponding $\Delta C_{t}$ values are listed in Supplementary Table 6. (b) Eleanor FISH signals were enlarged in LTED cells and diminished by resveratrol treatment (LTED-RES). The BAC probes covered the ESR1 locus (ESR1-BAC), a centromeric region of human chromosome 6 (CEN6-BAC), exons only (ESR1-cDNA) and the intron only (ESR1-intron 2, see Fig. 1e, bottom). Cellular DNA was heat-denatured in the top panels, but not in others. Eleanor foci were enlarged in LTED cells, which were detected with ESR1-BAC and intron probes, but not the ESR1-cDNA probe. Box plots on the bottom right show quantification of FISH signals in the top panels ( $n>170$ nuclei for each sample). $P$-values were calculated using Student's $t$-test ( $\left.{ }^{\star \star \star} P<0.001\right)$. Scale bar, $10 \mu \mathrm{m}$. (c) Sequential hybridization to Eleanor ncRNAs (RNA FISH, left) and the ESR1 gene (DNA FISH, middle) showing co-localization (right). Cellular RNAs were digested with RNase before DNA hybridization. Scale bar, $5 \mu \mathrm{m}$. (d) Gene tracks representing mRNA-Seq and RNA-Seq data of the ERBB2 locus in MCF7 and LTED cells. Compared with the ESR1 locus (Fig. 1e), there was no induction of ncRNAs in the ERBB2 locus of LTED cells. (e) Comparable induction levels of ESR1 and ERBB2 mRNAs in LTED cells. qRT-PCR results under the MCF7 condition were set to 1 . Values are the means \pm s.d.; $n=3$. Corresponding $\Delta C_{t}$ values are listed in Supplementary Table 6 . (f) Representative FISH images using BAC probes for the ESR1 locus (red) and ERBB2 locus (green). ERBB2 signals were not enlarged in LTED cells. Scale bar, $10 \mu \mathrm{m}$. (g) Box plot showing quantification of FISH data in $\mathbf{f}$ ( $n>100$ nuclei for each). 2M, two months; $4 \mathrm{M}$, four months. 
functions as an enhancer ${ }^{40,41}$. On the other hand, promoter A was high in $\mathrm{H} 3 \mathrm{~K} 4 \mathrm{me} 3$ relative to $\mathrm{H} 3 \mathrm{~K} 4 \mathrm{mel}$. The aligned ChIP-Seq data showed that the u-Eleanor region was also bound by CTCF, an organizer for the three-dimensional structure of the genome. Chromosome conformation capture experiments suggested that the $u$-Eleanor region as well as exon 1 and intron 1 of the ESR 1 gene were close together in LTED cells compared with that in MCF7 and LTED-RES cells (Supplementary Fig. 4). These results suggest that the upstream chromatin structure of this locus is significantly altered during LTED adaptation.

We tested whether $u$-Eleanor is involved in transcriptional activation of the ESR1 locus in LTED cells. Upon reduction of $u$-Eleanor to $50-70 \%$ by two independent siRNAs, ESR1 mRNA expression was decreased to $\sim 60 \%(P<0.01, P$-values; Student's $t$-test ) without affecting ERBB2 mRNA (Fig. 4d). Consistently, the ER protein level was decreased with the reduction of u-Eleanor (Fig. 4e). Furthermore, FISH analyses of LTED cells were performed using ESR1-BAC and ESR1-BAC2 (shown in Fig. 1e) as independent probes (Fig. 4f). u-Eleanor knockdown efficiently diminished the enlarged FISH signals, indicating that $u$-Eleanor maintains downstream intragenic Eleanor in LTED cells.

Next, we examined the effect of Eleanors on the proliferative activity of LTED cells. As a result, the cell number was significantly reduced at $96 \mathrm{~h}$ after knockdown of $u$-Eleanor by siRNA (Fig. 4g). Thus, $u$-Eleanor has an essential role in the enhanced transcription of Eleanors and mRNA from the ESR1 locus, as well as the cell proliferation and viability during LTED adaptation.

Resveratrol exerts a repressive effect on Eleanor via ER. As described above, addition of resveratrol to LTED cells dramatically co-suppressed the expression of both Eleanors and ESR1 mRNA (Figs 1b,e and $4 \mathrm{~b}$, red bars). As a result, resveratrol inhibited the proliferative activity of LTED cells in a dose- and time-dependent manner (Fig. 5a). Because oestrogen-loaded ER negatively regulates the ESR 1 gene ${ }^{42}$, we expected that resveratrol would repress the ESR1 locus through ER. To test this hypothesis, we depleted ER with specific siRNAs in LTED cells followed by resveratrol treatment (RES, Fig. 5b). FISH analyses using the ESR1-BAC probe showed that the Eleanor-containing foci in control cells ( $\mathrm{siGL3}$ ) became smaller after resveratrol treatment, but they remained large in ER-depleted cells (siESR1, Fig. 5c,d). These results indicated that suppression of Eleanors by resveratrol is dependent on ER. To confirm this result, we used a specific ER antagonist, ICI 182,780, which induces degradation of ER through the ubiquitin-mediated pathway ${ }^{43}$. After ICI 182,780 treatment of LTED cells for $48 \mathrm{~h}$, ER was absent (Fig. 5e). Under this condition, the enlarged Eleanor FISH signals became insensitive to the suppressive action of resveratrol (Fig. 5f). Moreover, we found that ER degradation by ICI 182,780 in LTED-RES cells resulted in de-repression of $u$-Eleanor transcripts (Fig. 4b, blue bars), indicating that resveratrol inhibits $u$-Eleanor expression via oestrogenic effects on ER. This result may be explained by the presence of multiple oestrogen-response elements in the u-Eleanor region (Supplementary Fig. 5, marked with yellow). Because u-Eleanor is required for enhanced expression of intragenic Eleanor in LTED cells (Fig. 4f), it is possible that resveratrol represses $u$-Eleanor through ER, leading to a subsequent reduction of intragenic Eleanor. In addition, repression of $u$-Eleanor and Eleanors by resveratrol was abrogated by ICI 182,780, whereas ESR1 mRNA remained repressed under the same condition (Fig. 5g). Thus, Eleanors were expressed even under repression of the ESR1 gene,
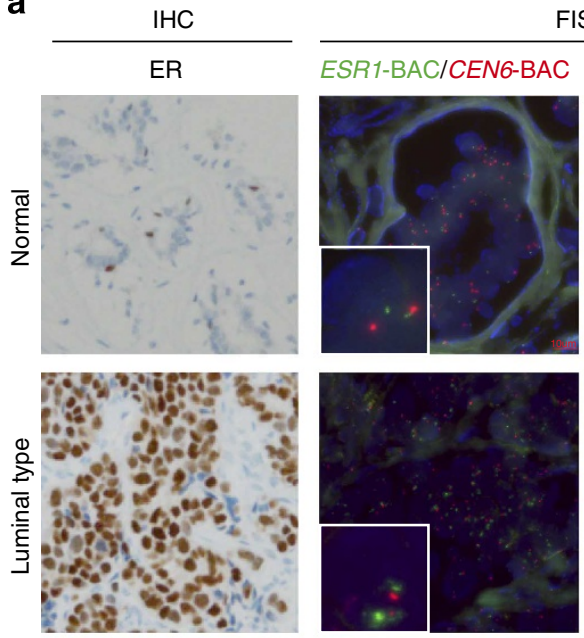

FISH
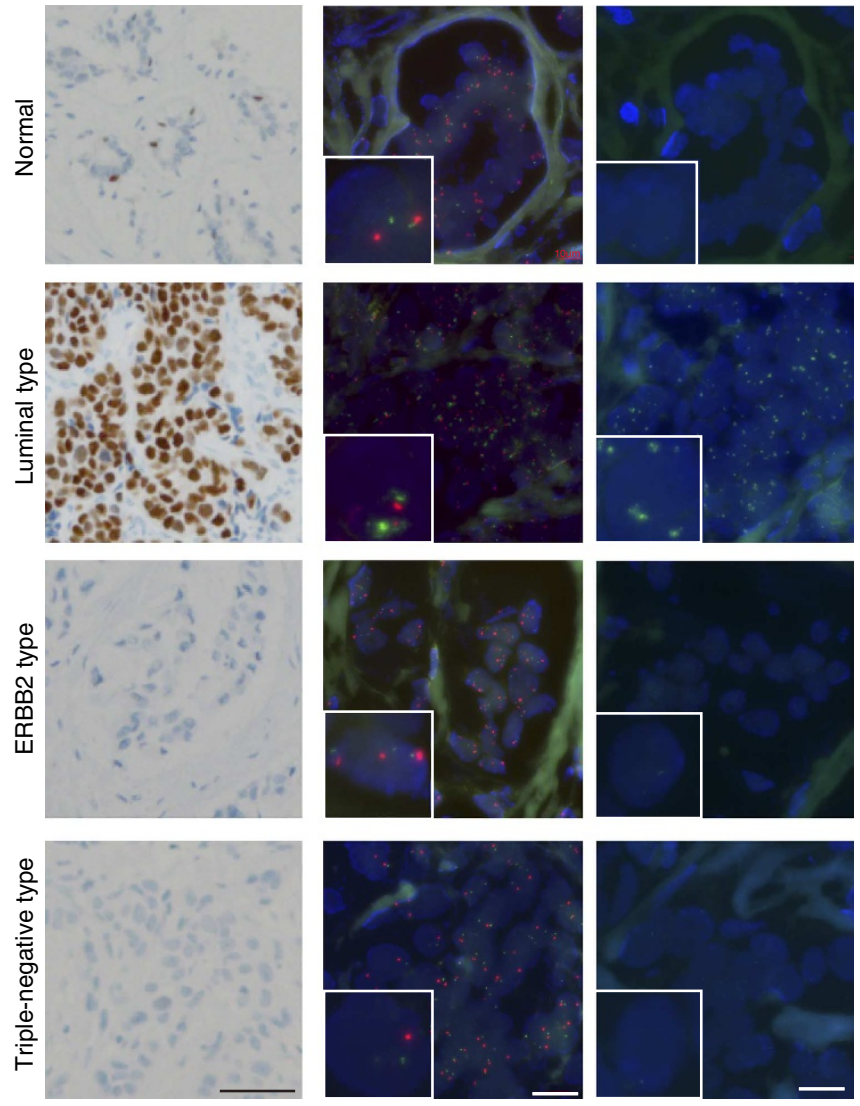

b

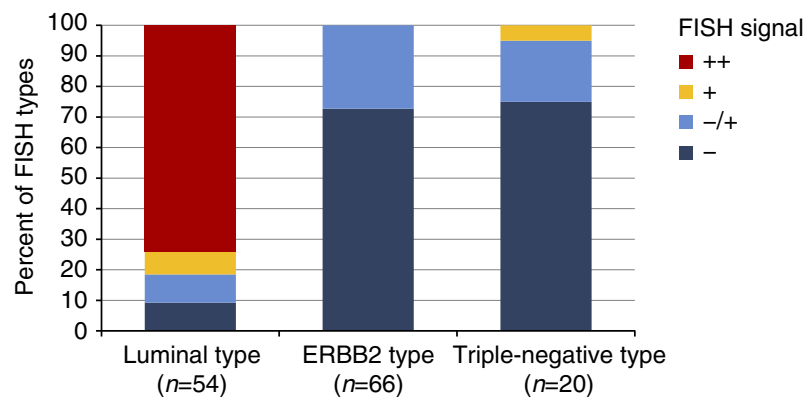

Figure 3 | Eleanor expression in ER-positive breast cancer tissues.

(a) IHC and FISH analyses of breast cancer tissues. Serial sections of breast cancer tissues with the indicated types were subjected to $\mathrm{IHC}$ using an anti-ER antibody (IHC, left) and FISH using BAC probes for ESR1 and CEN6 (middle and right, respectively). Large Eleanor-containing foci were detected in the luminal type (ER positive). The DNA was processed with or without heat denaturation (middle and right, respectively). Scale bars, $50 \mu \mathrm{m}$ (left) and $20 \mu \mathrm{m}$ (middle and right). (b) Summary of FISH analyses of breast cancer patients. Strong FISH signals $(++)$ were exclusively present in a subset of ER-expressing breast cancers (luminal type). Detailed data are provided in Supplementary Table 4.

indicating that Eleanors do not simply represent nascent transcripts or by-products of ESR1 mRNA.

Resveratrol has been reported to activate SIRT1, a member of the sirtuin family of $\mathrm{NAD}^{+}$-dependent deacetylases. Therefore, we examined whether SIRT1 is involved in the repressive effect of resveratrol on Eleanors. We depleted SIRT1 by siRNAs in LTED cells (Supplementary Fig. 6a,b) and then visualized Eleanor- 
a
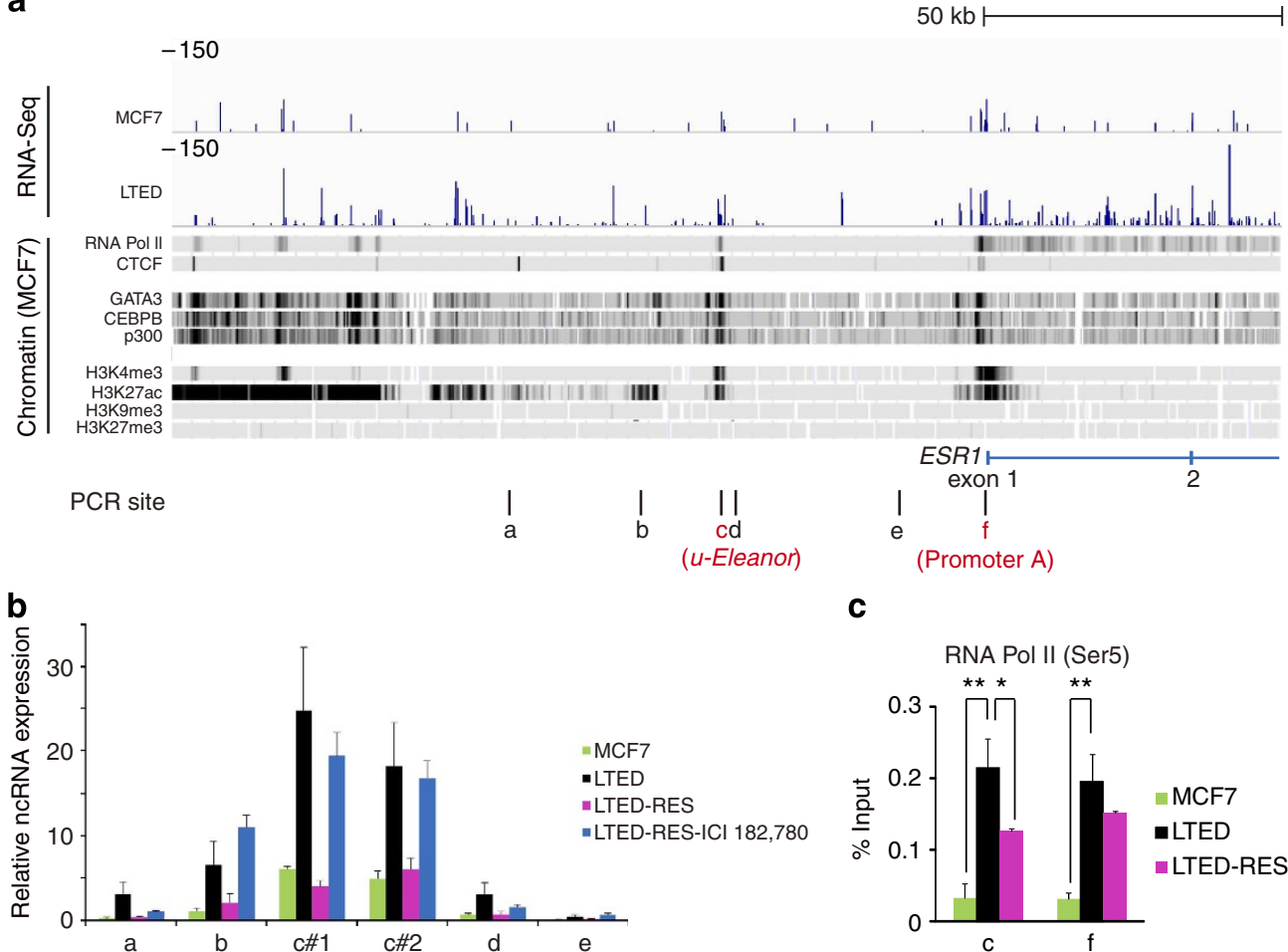

d
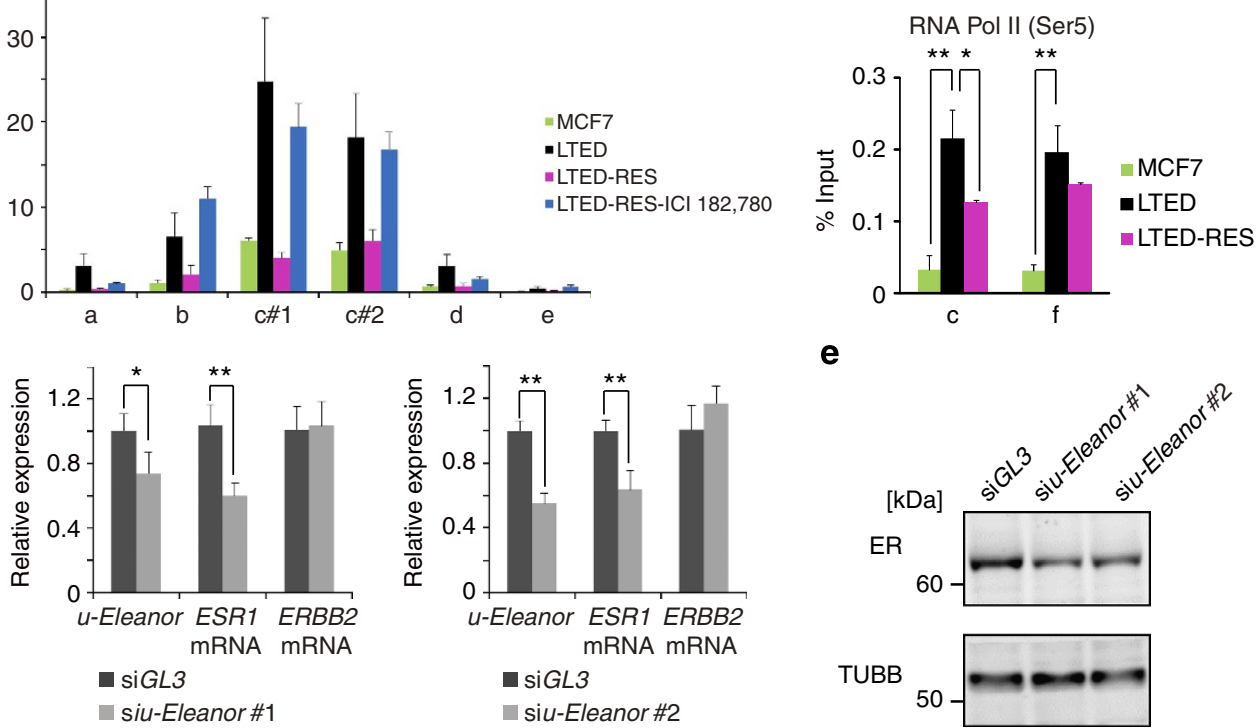

e

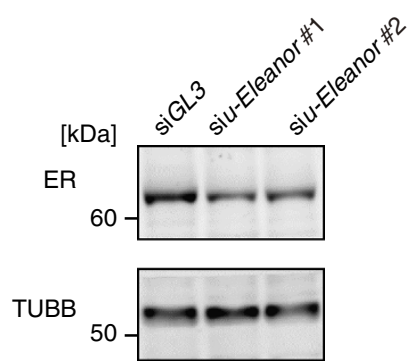

f

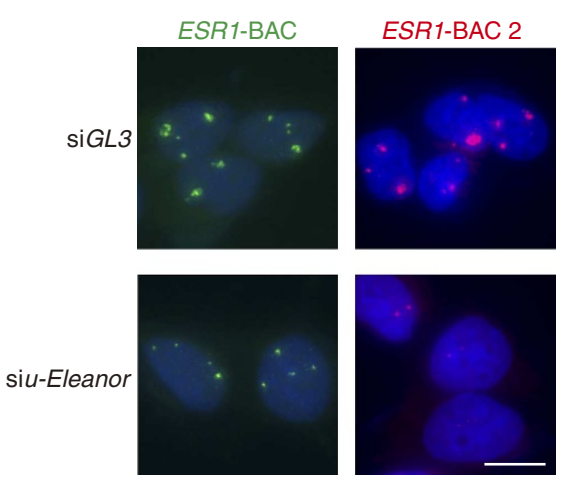

$\mathbf{g}$

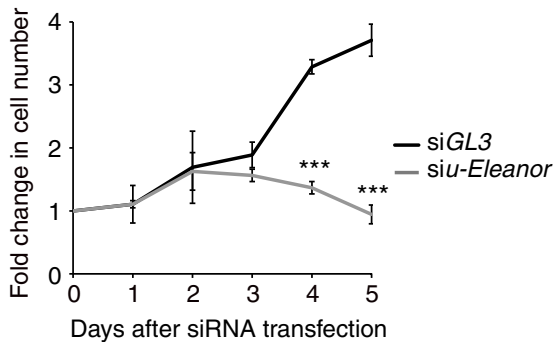

Figure 4 | $u$-Eleanor plays a role in coordinated up-regulation of intragenic Eleanor and ESR1 mRNA to promote the proliferative activity of LTED cells. (a) Overview of a region upstream of the ESR1 locus. The RNA-Seq tracks were aligned with the ChIP-Seq data available in the UCSC genome browser ${ }^{39}$ (University of California, Santa Cruz, CA, and Supplementary Table 1). Sites amplified by qPCR are shown (a-f). (b) Local expression of $u$-Eleanor. u-Eleanor was induced at site $\mathrm{c}$ in LTED cells, repressed in LTED-RES cells, and de-repressed by ICI 182,780 treatment (ER antagonist, related to Fig. 5e-g).

For qRT-PCR, total RNA was pre-treated with DNase I, and the amplification efficiency for each primer set was normalized. The value for site $b$ in MCF7 was set to 1. Values are the means \pm s.d.; $n=3$. Corresponding $\Delta C_{t}$ values are listed in Supplementary Table 6. (c) RNA Pol II binding to the $u$-Eleanor region (c) and ESR1 promoter A ( $f$ ). For ChIP-qPCR, values are the means \pm s.d.; $n=3$. $P$-values were calculated using Student's $t$-test ( $\left.{ }^{\star \star} P<0.01\right)$. (d) Decreased expression of ESR1 mRNA upon u-Eleanor knockdown by specific siRNAs. Expression levels of ESR1 and ERBB2 mRNAs were measured by qRT-PCR. Values are the means \pm s.d.; $n=3$. $P$-values were calculated using Student's $t$-test $\left({ }^{\star} P<0.05\right.$, ${ }^{\star \star} P<0.01$ ). (e) Reduced protein level of ER upon $u$-Eleanor knockdown. An immunoblot of ER is shown. TUBB served as a loading control. (f) Reduction of Eleanor-containing foci by $u$-Eleanor knockdown in LTED cells. Independent BAC probes shown in Fig. 1e were used for the FISH analyses. Scale bar, $10 \mu \mathrm{m}$. (g) Inhibition of LTED cell proliferation by u-Eleanor knockdown. LTED cells were treated with siRNA targeting $u$-Eleanor for the indicated periods. Cell numbers are shown as fold changes. Values are the means \pm s.d.; $n=3$. $P$-values were calculated using Student's $t$-test $\left({ }^{\star} P<0.05,{ }^{\star \star \star} P<0.001\right)$. 


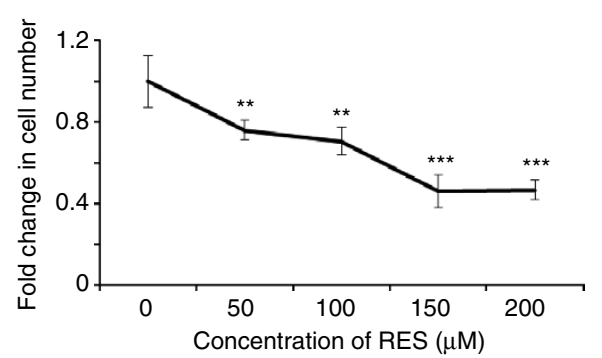

b

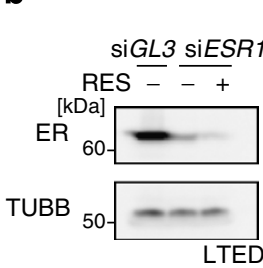

C
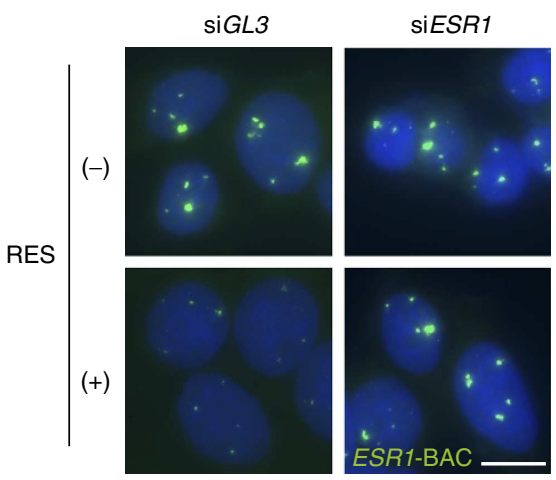

d

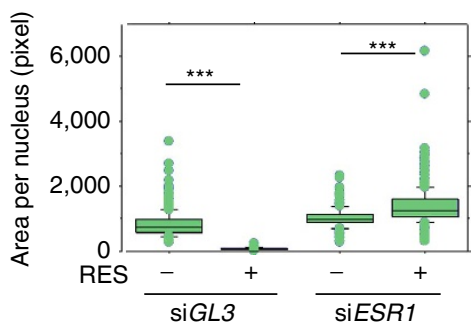

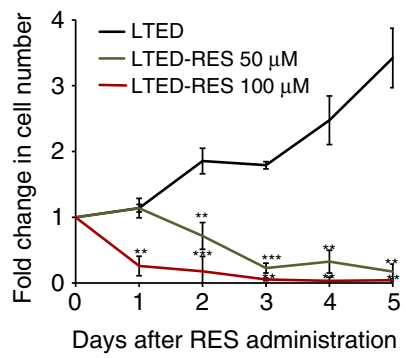

e

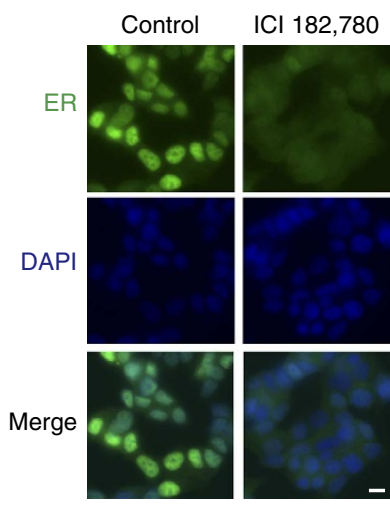

$\mathbf{f}$

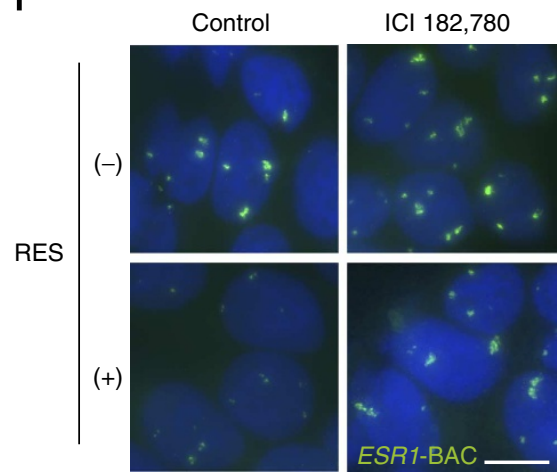

g

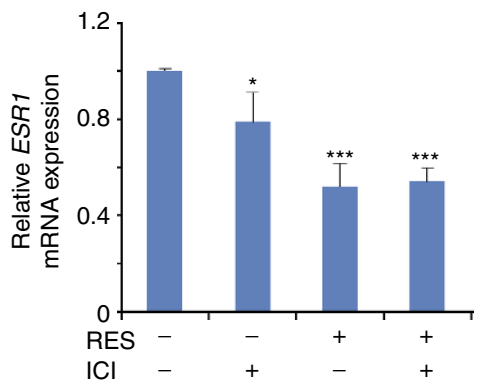

Figure 5 | Resveratrol exerts a repressive effect on Eleanors via ER. (a) Inhibition of LTED cell proliferation by resveratrol treatment. Cell proliferation was inhibited in a dose-dependent manner (left). The number of control LTED cells was set to 1 . A time course analysis was performed using 50 and $100 \mu \mathrm{M}$ resveratrol (right). The number of LTED cells at day 0 was set to 1 . Values are the means \pm s.d.; $n=3$. P-values were calculated using Student's $t$-test $\left({ }^{\star \star} P<0.01,{ }^{\star \star \star} P<0.001\right)$. (b) Immunoblot and immunofluorescence analyses of siRNA-mediated ER knockdown. Scale bar, $10 \mu \mathrm{m}$. (c) FISH images of Eleanor-containing foci (green) obtained with the ESRT-BAC probe in LTED cells. The reduction of Eleanor foci by resveratrol was dependent on ER. Scale bar, $10 \mu \mathrm{m}$. (d) Box plot showing quantification of the FISH signals in c. $n>200$ nuclei for each sample. P-values were calculated using Student's $t$-test ( $\left.{ }^{\star \star \star} P<0.001\right)$. (e) Immunofluorescence analysis of ER degradation in LTED cells treated with ICI 182,780 (100 nM) for $48 \mathrm{~h}$. Scale bar, $10 \mu \mathrm{m}$.

(f) ICI 182,780 treatment inhibited the reduction of Eleanor foci by resveratrol treatment. The results were similar to those obtained by ER knockdown in c. Scale bar, $10 \mu \mathrm{m}$. (g) qRT-PCR analyses of LTED cells showing repression of ESR1 mRNA by resveratrol treatment (RES + ). ICI 182,780 had no de-repressive effect (RES,$+ I C l+$ ). Values are the means \pm s.d.; $n=3$. $P$-values were calculated using Student's $t$-test $\left({ }^{\star} P<0.05\right.$, $\left.{ }^{\star \star \star} P<0.001\right)$. 
a

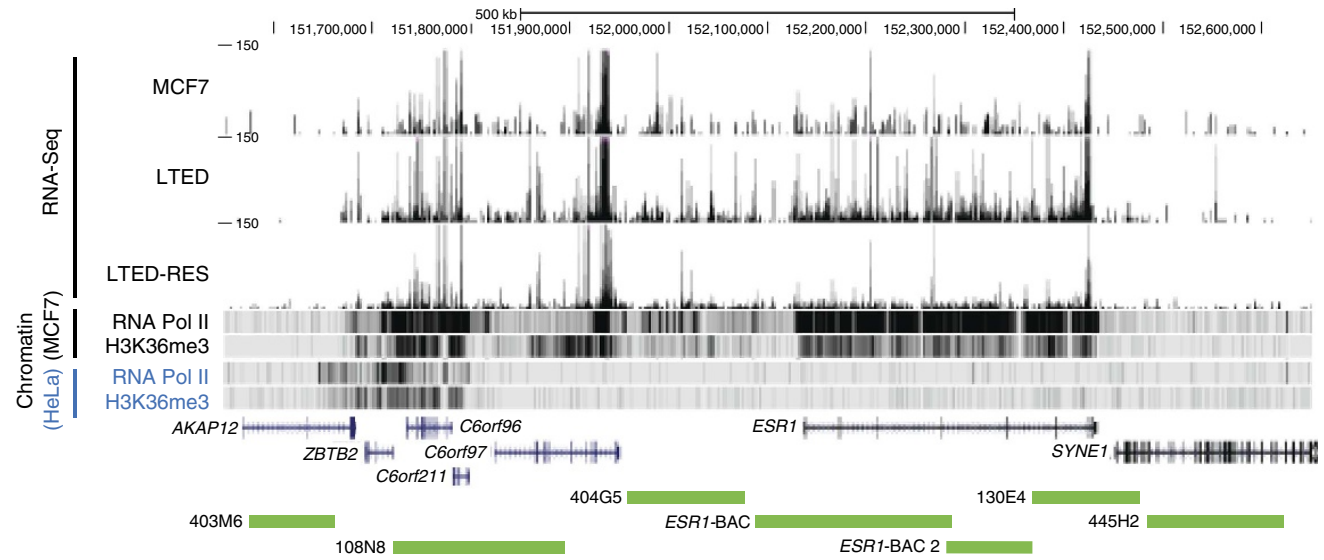

b
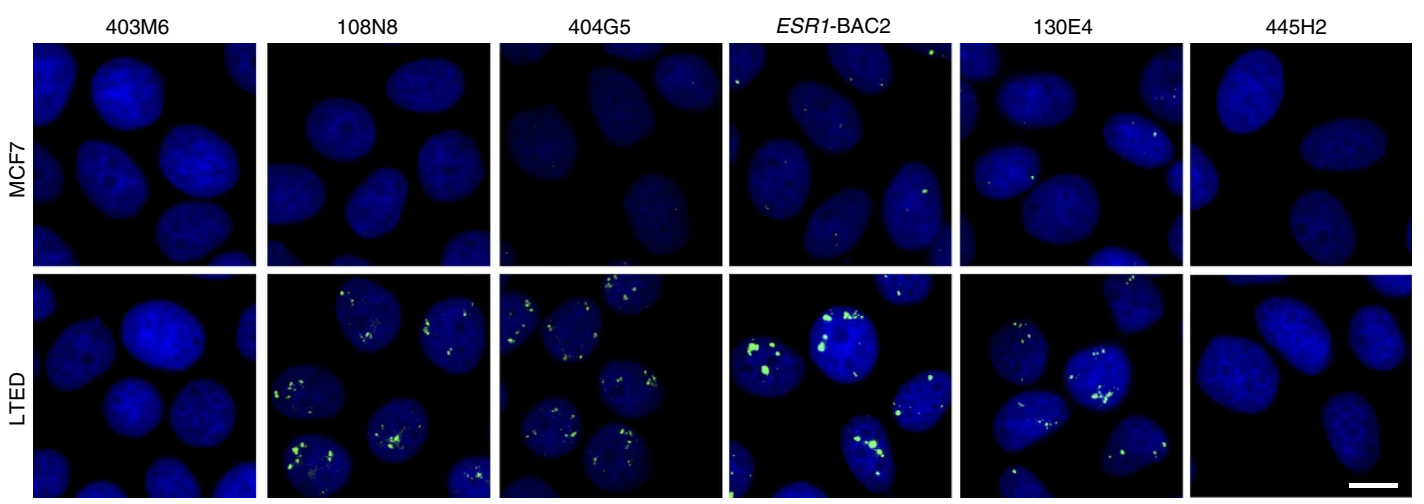

$\mathbf{C}$
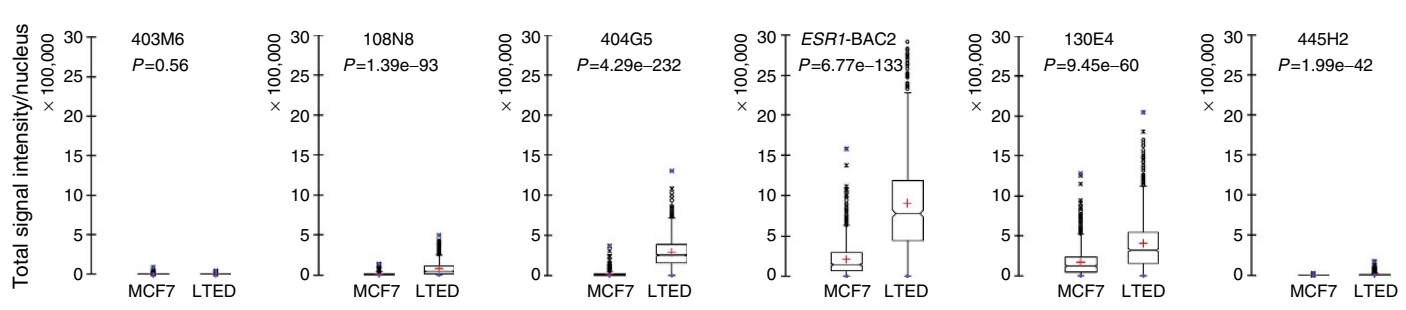

d
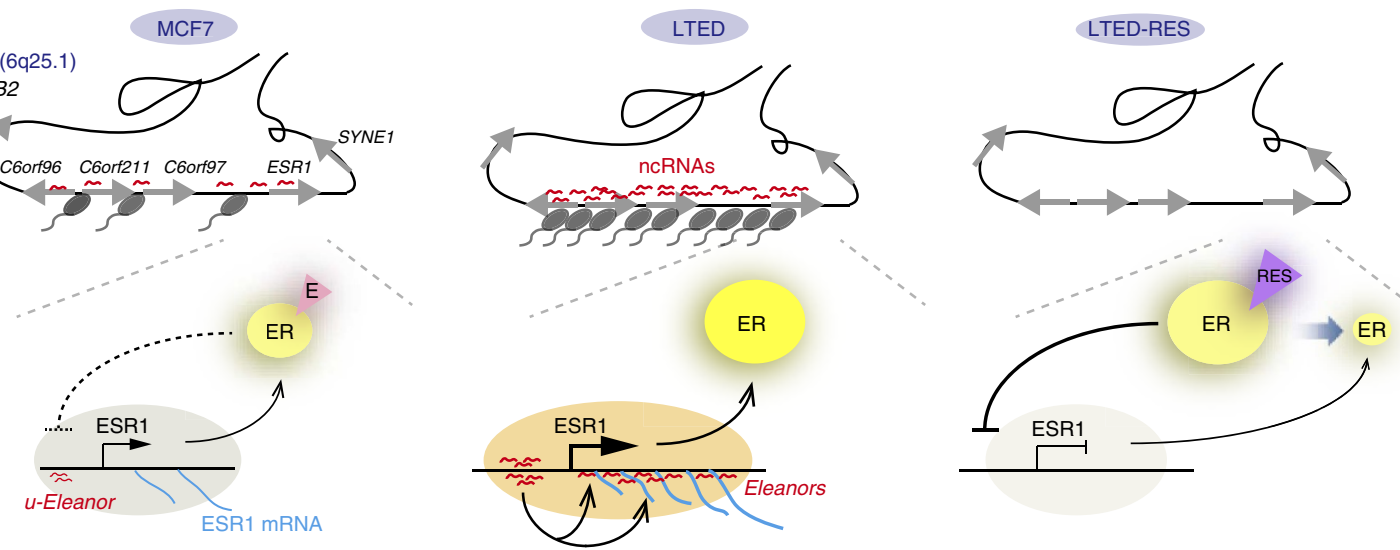

Figure 6 | Large chromatin domain is defined by production of ncRNAs in LTED cells. (a) Overview of the $\sim 1$ Mb region surrounding the ESR1 locus. The RNA-Seq tracks were aligned with the ChIP-Seq data available in the UCSC genome browser ${ }^{39}$ (Supplementary Table 1). In LTED cells, RNAs were abundantly expressed from a large region including C6orf96, C6orf211, C6orf97 and ESR1 genes (6q25.1; 151,720,000-152,424,447). Green bars indicate the BAC clones used for FISH experiments. (b) RNA-FISH scanning analyses along the region of 6q25.1. Scale bar, $10 \mu \mathrm{m}$. In LTED cells, nuclear RNA foci were detected with BAC clones 108N8, 404G5, ESR-BAC2 and 130E4. (c) Box plot showing quantitative analysis of RNA-FISH in b. Total signal intensities per nucleus in MCF7 and LTED cells ( $n=700 \sim 900$ nuclei per sample). (d) Schematic model for ER-positive breast cancer adaptation. Overviews of the chromatin domain including the ESR1 gene are at the top. RNA Pol II (a black oval with a tail) is bound to this region, and ncRNAs are transcribed at the basal level in MCF7 cells. In LTED cells, the ncRNAs are transcribed actively throughout the chromatin domain and stably associate with the site of transcription in LTED cells, leading to the formation of an RNA cloud in the nucleus. Resveratrol treatment suppresses the ncRNA production. Enlarged views of the ESR1 locus are shown at the bottom. 
containing foci by FISH. Similar to the control (siGL3), Eleanorcontaining signals became smaller after resveratrol treatment under SIRT1 knockdown, suggesting that resveratrol represses Eleanors in the absence of SIRT1 (Supplementary Fig. 6c). In addition, overexpression of SIRT1 in LTED cells did not change the FISH signals (Supplementary Fig. 6d). Thus, the effect of resveratrol on the ESR1 locus is unlikely to depend on SIRT1.

A large chromatin domain is defined by a cluster of ncRNAs. An overview of the RNA-Seq data around the ESR1 gene revealed that the region associated with ncRNAs spanned $\sim 700 \mathrm{~kb}$ (6q25.1; 151,720,000-152,424,447; Fig. 6a). In addition to ESR1, this region includes three previously annotated genes, C6orf96, C6orf211 and C6orf97, all of which are co-regulated in breast cancer cells ${ }^{44}$. Alignment with the published ChIP-Seq data revealed that the region was heavily bound by RNA Pol II and enriched with an active histone mark, trimethyl-H3K36, in breast cancer cell lines, but not other cell types such as HeLa (Fig. 6a). The length of the region was well correlated with the recently proposed size for a single unit of a chromatin domain ${ }^{19-21}$.

To visualize characteristic RNAs originating from this large region, we performed RNA-FISH scanning using a series of BAC probes for subregions along the chromatin domain (Fig. 6a,b). We detected large RNA foci with probes 108N8, 404G5 and $130 \mathrm{E} 4$, which were similar to those detected with ESR1-BAC and
ESR1-BAC2 probes (Figs $6 \mathrm{~b}, \mathrm{c}$ and $2 \mathrm{~b}$ ). It should be noted that $404 \mathrm{G} 5$, which corresponded to a region completely devoid of any protein-coding sequences, was able to detect the large RNA foci. In contrast, RNAs were not produced from outside of the domain (403M6 and 445H2). Using the combination of RNA-Seq and RNA-FISH, we found a novel chromatin domain including four co-regulated genes that were defined by induction of a ncRNA cluster during hormone deprivation (see Fig. 6d).

A set of long genes exhibit co-regulation of ncRNA and mRNA. Our genome-wide transcriptome analyses revealed changes in the mRNA expression of 2,918 genes under MCF7, LTED and LTEDRES conditions (interquartile range $>10$ ). They were classified into 14 distinct clusters on the basis of expression patterns (Supplementary Fig. 7a). Among them, genes in cluster 4 (199 genes including ESR1) showed induction in LTED cells and repression in LTED-RES cells (Fig. 7a). Gene ontology analysis of cluster 4 showed enrichment of genes for the cellular metabolic process, transcriptional regulation, apoptosis and cell death, and cellular response to oestrogen stimulus (Fig. 7b).

We further characterized ncRNAs that were co-regulated with their neighbouring protein-coding genes. RNA-Seq analyses showed that $\sim 168,000$ gene regions were significantly up- or down-regulated during LTED adaptation (MCF7 to LTED) and resveratrol treatment (LTED to LTED-RES, Fig. 7c). Interestingly, a

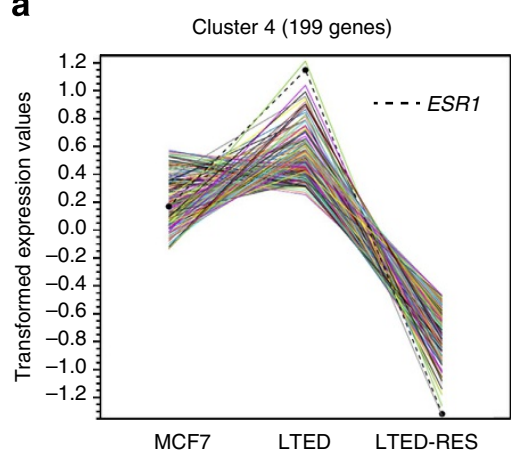

b

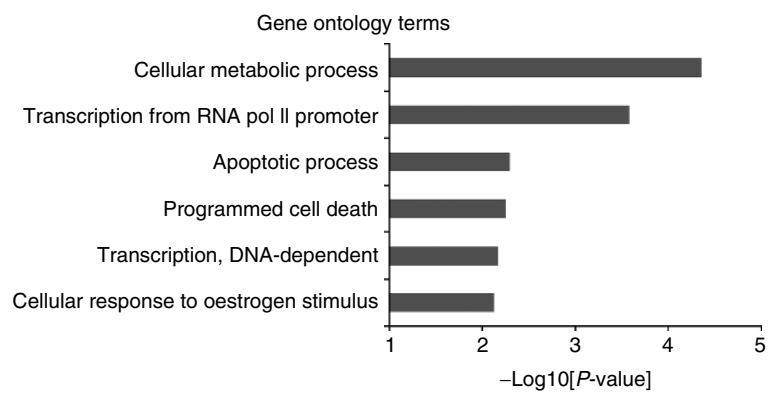

C

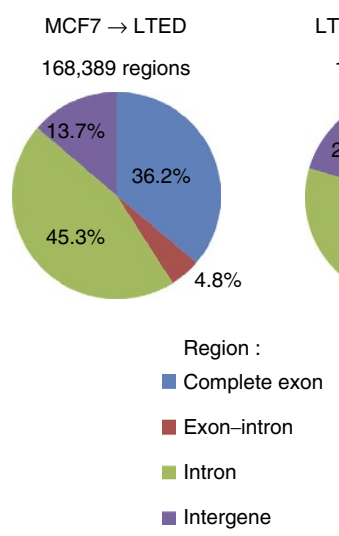

d

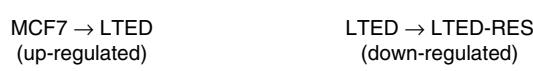

168,749 regions

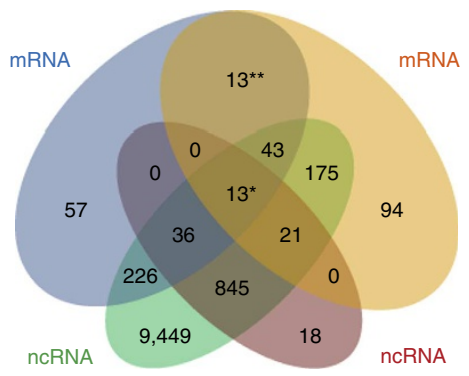

Figure 7 | Genome-wide transcriptome analyses of LTED adaptation and resveratrol treatment. (a) Expression patterns of mRNAs from 199 genes in cluster 4. ESR1 was the most dynamically up-regulated in LTED cells and down-regulated in LTED-RES cells. (b) Gene ontology terms significantly enriched in cluster 4. (c) Summary of RNA-Seq data in LTED adaptation (MCF7 to LTED) and resveratrol treatment (LTED to LTED-RES). Differentially expressed RNAs (log2 fold change $<-1.0$ or $>1.0, P<0.01$ ) were classified into four groups: complete exon, exon-intron, intron and intergene, as described in the Methods. P-value was calculated with edgeR ${ }^{68}$. The percentages of ncRNAs, excluding complete exons, were $63.8 \%$ (left) and 67.3\% (right). (d) Genes with coordinate regulation of ncRNAs and mRNAs. Comparison of mRNA-Seq and ncRNA-Seq data resulted in the extraction of 13 gene regions (single asterisk) that showed simultaneous up-regulation of mRNAs and ncRNAs under MCF7 to LTED conditions and subsequent down-regulation under LTED to LTED-RES conditions ( $\log 2$ fold change $<-1.0$ or $>1.0, P<0.01$ ). P-value was calculated with DESeq and edgeR 67,68 . The other 13 genes (double asterisks) also showed up- or down-regulation of mRNAs in LTED and LTED-RES cells, respectively, while ncRNAs showed no co-regulation. The genes in these groups and their gene lengths are listed in Table 1. 


\begin{tabular}{|c|c|c|c|}
\hline \multicolumn{2}{|c|}{$\begin{array}{l}\text { Genes with coordinated } \\
\text { transcription }\end{array}$} & \multicolumn{2}{|c|}{$\begin{array}{c}\text { Genes without coordinated } \\
\text { transcription }\end{array}$} \\
\hline Gene symbol & Gene length (bp) & Gene symbol & Gene length (bp) \\
\hline SDK1 & 967,552 & $E R B B 2$ & 40,523 \\
\hline$\angle R B A$ & 750,839 & $B R D 8$ & 38,900 \\
\hline RERE & 465,236 & SLC12A5 & 38,461 \\
\hline ESR1 & 412,778 & CLN3 & 25,430 \\
\hline USP34 & 283,260 & SIDT2 & 18,223 \\
\hline SYNPO2 & 210,560 & DBNDD1 & 14,659 \\
\hline KDMA2A & 138,811 & CASP14 & 8,813 \\
\hline KYNU & 111,912 & ESRP2 & 7,687 \\
\hline AVL9 & 88,604 & TUBG2 & 7,759 \\
\hline CCDC50 & 68,586 & CYP1A1 & 5,995 \\
\hline SYTL2 & 63,780 & VPS28 & 4,963 \\
\hline AHNAK & 41,104 & APH1A & 3,811 \\
\hline ATIC & 37,818 & MFSD10 & 3,677 \\
\hline Average length & 280,142 & Average length & 16,839 \\
\hline
\end{tabular}

*Genes that showed simultaneous up-regulation of mRNAs and ncRNAs under MCF7 to LTED conditions and subsequent down-regulation under LTED to LTED-RES conditions (denoted with single asterisk in Fig. 7d).

$\dagger$ Genes that showed up- and down-regulation of mRNAs in LTED and LTED-RES cells,

respectively, while ncRNAs showed no co-regulation (denoted with double asterisks in Fig. 7d).

over $60 \%$ of these gene regions corresponded to potential ncRNAs, including exon-intron fusion, intron and intergenic sequences. We then extracted gene regions in which both mRNAs and ncRNAs were up-regulated during LTED adaptation and down-regulated by resveratrol treatment (Fig. 7d). Thirteen gene regions (indicated with a single asterisk in Fig. $7 \mathrm{~d}$ and Table 1), including the ESR1 locus, exhibited such coordinate expression in LTED and LTED-RES cells (Fig. 7d and Supplementary Fig. 7b). We found that the genes in this group were generally long. Their average length was close to $300 \mathrm{~kb}(280,142 \mathrm{bp})$, while the average for genes in the overall genome is $\sim 60 \mathrm{~kb}$ (ref. 45 ). In the other 13 gene regions (indicated with double asterisks in Fig. $7 \mathrm{~d}$ and Table 1), similar to the ESR1 locus, mRNAs were up- and downregulated in LTED and LTED-RES cells, respectively. However, they lacked coordinated regulation with neighbouring ncRNAs (Fig. 7d and Supplementary Fig. 7c). Their average length was $\sim 17 \mathrm{~kb}(16,839 \mathrm{bp})$.

Thus, our comprehensive transcriptome analyses revealed that coordinated regulation of ncRNA and mRNA, as exemplified by Eleanors and ESR1 mRNA, is common in a set of long genes. On the basis of recent studies ${ }^{45-47}$, ncRNA production from the entire gene locus may overcome physical transcription complications and maintain open chromatin.

\section{Discussion}

In this study, we found a novel type of ncRNA-mediated gene locus control in breast cancer adaptation. While cells undergo hormone-independent proliferation, the ESR1 gene is upregulated and ncRNAs are produced from a broad chromatin domain of $\sim 700 \mathrm{~kb}$ including ESR 1 and other co-regulated genes. Eleanors originate from and around the ESR1 gene, and maintain the transcriptionally active locus. Up-regulation of ESR1 is important for the hormone-independent cell growth, which is suppressed by inhibition of Eleanors with either u-Eleanor knockdown or resveratrol. Eleanors are overexpressed in ER-positive breast cancers, suggesting that resveratrol and an inhibitor of $u$-Eleanor may be potential therapeutic agents for endocrine therapy resistance.

On the basis of our data, we propose a mechanistic model as illustrated in Fig. 6d. ESR1 mRNA is expressed at the basal level in MCF7 cells as they are ER positive (Fig. 6d, MCF7). During LTED adaptation, because of the loss of negative control by oestrogen-bound ER, there is significant induction of $u$-Eleanor expression, leading to coordinate up-regulation of intragenic Eleanor and ESR1 mRNA (Fig. 6d, LTED). Characteristic Eleanor-containing RNA foci are formed in the nuclei of LTED cells and subsequent resveratrol treatment represses both $u$-Eleanor and intragenic Eleanor by the oestrogenic actions on ER (Fig. 6d, LTED-RES).

The mechanism for induction of $u$-Eleanor in LTED cells is intriguing, and one possible mechanism is de-repression. In MCF7 cells, oestrogen-bound ER mildly inhibits the ESR1 locus by negative feedback ${ }^{48}$. On removal of oestrogen, ER becomes unliganded and fails to repress the ESR 1 locus. By the addition of resveratrol to LTED cells, resveratrol-bound ER strongly represses the ESR1 locus, possibly because of its high concentration or structural properties.

Cancer cells can survive during various environmental changes by adjusting their global gene expression to acquire suitable phenotypes. Indeed, our transcriptome analyses of MCF7 and LTED cells showed that 2918 mRNAs were significantly up- or down-regulated under oestrogen deprivation (Supplementary Fig. 7a). These transcriptional changes were not limited to protein-coding regions (Fig. 7c, complete exon) and rather prevalent ( $\sim 2$-fold more frequent) in noncoding regions (Fig. $7 \mathrm{c}$, exon-intron, intron and intergene). These results are in good agreement with the fact that $<2 \%$ of the mammalian genome encodes proteins, whereas $75 \%$ of the genome is transcribed to produce ncRNAs that may modulate chromatin structure and gene expression ${ }^{26,49}$.

For the following reasons, Eleanors identified in this study are unique. First, transcription of Eleanors is inducible. They accumulate during oestrogen deprivation, before ESR1 mRNA up-regulation, and are abruptly suppressed by resveratrol. Furthermore, genome-wide analyses revealed that the ESR1 locus is one of the 13 genetic loci where ncRNAs and mRNAs are co-regulated during the hormonal changes.

Second, intragenic Eleanors are transcribed from a broad region covering the entire ESR1 gene body, which spans up to $300 \mathrm{~kb}$. In addition, $u$-Eleanor is derived from an enhancer-like element at $\sim 40 \mathrm{~kb}$ upstream of the ESR1 gene. u-Eleanor is responsible for up-regulation of downstream transcripts including ESR1 mRNA and intragenic Eleanors (Fig. 4d,f). To date, there are few reports of simultaneous transcription of ncRNAs from upstream and the gene body. One related but distinct example is the $\beta$-globin locus where ncRNAs are produced from LCR, a central upstream regulatory element, and downstream intergenic regions in the locus ${ }^{50}$.

Third, Eleanors cover their own transcription sites to regulate gene expression and form a so-called 'RNA cloud' in the nucleus (Fig. 2c). This property may be shared with other chromosomal RNAs including XACT, Air, $\mathrm{C}_{0} \mathrm{~T}-1$ repeat RNAs and snoRNAs ${ }^{51-54}$. XIST RNA also forms a large nuclear domain by coating the entire inactive $\mathrm{X}$ chromosome and plays a role in $\mathrm{X}$ chromosome inactivation ${ }^{55}$. Eleanors may be functional introns that are stably maintained in the nucleus ${ }^{56-58}$ or represent pervasive transcription of the genome $\mathrm{e}^{59}$, which are enhanced under oestrogen deprivation. Eleanors were unusually stable because of resistance to the denaturation procedure that is normally performed only in DNA FISH and degrades most RNAs. Because the Eleanor FISH signals were clearer with denaturation (Fig. 2b, top panels), Eleanors may be tethered to the sites of transcription by RNA-DNA hybrid formations.

We found that coordinate expression of ncRNA and mRNA is conserved in a set of long genes (Fig. 7, Table 1). Accumulation of ncRNA at the site of transcription may counteract length- 
dependent impairment of gene transcription that was reported recently ${ }^{45,47}$. We and others have previously reported that a population of ER-positive breast cancer cells has abnormally large ESR1 FISH signals ${ }^{60-62}$. Considering that Eleanors are extremely stable, it is possible that some of the previously detected large FISH signals in breast cancer patients include Eleanors as shown in Fig. 3. In addition, several molecular events have been reported during LTED adaptation, including up-regulation of ER, ERBB2, c-Myb, c-Myc and MAP kinases, and activation of the PI-3 kinase pathway, NOTCH pathway, growth factor pathways related to mTOR and EGFR/ERBB/AKT, as well as changes in the phosphorylation pattern of $\mathrm{ER}^{7,8,10,31,63}$. It would be interesting to determine how Eleanors are integrated in these events in LTED cells and ER-positive breast cancer cells.

In summary, Eleanors, a novel type of ncRNA, are actively involved in the epigenetic adaptation of ER-positive breast cancer cells by activating transcription of the ESR1 gene locus. These findings highlight ncRNA-mediated mechanisms in cancer cell adaptation, which may be diagnostic and therapeutic targets for endocrine therapy-resistant breast cancer.

\section{Methods}

Cell culture. MCF7 cells (ATCC) were cultured in RPMI 1640 (Sigma) supplemented with $10 \%$ fetal bovine serum (FBS). For LTED, MCF7 cells were grown in phenol red-free RPMI 1640 (Wako) containing 4\% dextran-coated charcoalstripped FBS for 2-4 months. For LTED-RES, LTED cells were treated with 50 or $100 \mu \mathrm{M}$ resveratrol (Sigma Aldrich, R5010) for $24 \mathrm{~h}$. For ICI 782,780 treatment, cells were cultured with $100 \mathrm{nM}$ ICI 782,780 (Tocris, 1047) for $48 \mathrm{~h}$. Human mammary epithelial cells ${ }^{64}$ (Lonza) were cultured in mammary epithelial growth media (Lonza) according to manufacturer's protocol at $37^{\circ} \mathrm{C}$ with $5 \% \mathrm{CO}_{2}$. Primary invasive breast carcinoma specimens were obtained by surgical excision from patients at the Department of Breast and Endocrine Surgery, Kumamoto University Hospital (Kumamoto, Japan).Informed written consent was obtained from all the patients before surgery. The study protocol was approved by the Ethics Committee of Kumamoto University Graduate School of Medicine (Kumamoto, Japan).

Antibodies. The following primary antibodies were used: rabbit polyclonal antihuman ER $\alpha$ (Santa Cruz Biotechnology, sc-543; dilution used in IB: 1:1,000, IF: 1:300), rabbit monoclonal anti-human ER $\alpha$ clone SP1 (Ventana, 790-4325; used in IHC without dilution), mouse monoclonal anti-human $\beta$-tubulin (Sigma Aldrich, T4026; dilution used in IB: 1:1,000), anti-RNA polymerase II (phosphor-S5) (Abcam, ab5131; dilution used in ChIP: 1:200), histone H3K4me1 (Abcam, ab8895; dilution used in ChIP: 1:200), histone H3K4me3 (Millipore, 07-473; dilution used in ChIP: 1:200), rabbit IgG (Santa Cruz Biotechnology, sc-2027; dilution used in ChIP: 1:100), rabbit polyclonal anti-SIRT1 (Millipore, 07-131; dilution used in IB: 1:1,000, IF: 1: 200), and mouse monoclonal anti-myc (Roche, clone 9E10 dilution used in IB: 1:1,000, IF: 1:100). The secondary antibodies used were Alexa Fluor 488-conjugated donkey anti-rabbit IgG (Molecular Probes; dilution used in IF: 1:300) and Cy3-conjugated donkey anti-mouse IgG (Jackson ImmunoResearch; dilution used in IF: 1:1,000). FITC-anti-digoxigenin (Roche; dilution used in FISH: 1:250) or Cy3-streptavidin (Jackson ImmunoResearch; dilution used in FISH: $1: 1,000)$.

BAC clones. For FISH analysis, we used BAC probes covering the ESR1 locus and flanking regions (RP11-403M6, RP11-108N8, RP3-404G5, RP11-450E24 (for ESR1-BAC), RP1-63I5 (for ESR1-BAC2), RP1-130E4 and RP3-445H2) and a probe containing the ERBB2 locus (RP11-94L15). To verify primers used in the 3C assay, we used BAC clones spanning the ESR1 locus (RP11-450E24 and RP3-404G5).

Preparation of mRNA-Seq libraries. Total RNA was extracted from cultured cells with an RNeasy Mini Kit (Qiagen). mRNA-Seq libraries were generated using an mRNA-Seq Sample Preparation Kit (Illumina) according to manufacturer's protocol with minor modifications. Poly(A) ${ }^{+}$RNA was enriched from $1 \mu \mathrm{g}$ total RNA by two successive rounds of oligo(dT) selection, fragmented and then used for first-strand cDNA synthesis by random hexamer priming. After second-strand cDNA synthesis, double-stranded DNA was repaired using T4 DNA polymerase, Klenow enzyme and T4 polynucleotide kinase (New England Biolabs), and then treated with Klenow exo- to add an adenine to the $3^{\prime}$ end. After ligation of the Index PE Adaptor oligo mix (Illumina) using Takara ligation mix (Takara), the adaptor-ligated DNA was amplified using primers, InPE2.0/1.0 and index (Illumina), by 18 cycles of PCR. The amplified libraries were isolated from an agarose gel. The DNA samples were purified using a QIAquick MinElute Kit (Qiagen) at each preparation step.
Preparation of RNA-Seq libraries. Total RNA was extracted from cultured cells with an RNeasy Mini Kit and then ribosomal RNA was removed by a Ribo-Zero Gold Kit (Illumina). RNA-Seq libraries were generated using a ScriptSeq v2 RNA-Seq Library Preparation Kit (Illumina), according to manufacturer's instructions.

mRNA-Seq and RNA-Seq analyses. An Illumina Genome Analyzer IIx was used to generate 41-base single-end reads for mRNA-Seq and an Illumina HiSeq 1000 was used to generate 51 base (stranded) single-end reads for RNA-Seq. The sequence reads were aligned with the human reference genome (UCSC hg19, http://genome.ucsc.edu/) using Tophat (v1.4.1, ref. 65), and RNA transcripts were reconstructed with Cufflinks (v1.3.0, ref. 66) with all parameters set to default values. The numbers of total and mapped reads are summarized in Supplementary Table 2.

Clustering of sequence reads and gene ontology. Data for mRNA-Seq experiments were analysed using CLC Genomics Workbench ver 5.51 (CLC Bio). Results obtained from mRNA-Seq experiments were selected on the basis of the following parameters: degree of variance in gene expression (inter quantile range $>10$ ) in two samples among MCF7, LTED and LTED-RES cells, and sufficient abundance of mRNA (FPKM >5). A total of 2,918 genes were chosen and used for downstream analyses including k-means clustering (Mev (http://www/tm4.org/men/)). In addition, cluster 4 (199 genes) obtained by k-means clustering was used for gene ontology analysis that was performed using the Genomatix Pathway System in the Genomatix Genome Analyzer. The distribution of reads in the RNA-Seq data set was determined by counting the number of reads in a 100-bp sliding window by the NGS Analyzer of the Genomatix Genome Analyzer and selected on the basis of the following parameters: differentially expressed transcripts in two different cell states (MCF7 and LTED, or LTED and LTED-RES) by fold-change ranking ( $\log 2$ fold-change $<-1.0$, down-regulation; $>1.0$, up-regulation) together with $P$-values $(P<0.01)$ computing by method described in chapter 'Testing for differential expression' in DEseq algorithm version 1.0.6 (ref. 67) in the NGS Analyzer of the Genomatix Genome Analyzer. This analysis identified 168,389 and 168,749 genetic regions that were differentially expressed in LTED cells compared with that in MCF7 cells, and LTED-RES cells compared with that in LTED cells, respectively. Furthermore, each region was subdivided by ElDorado database genome annotation version $08-2011$.

Combined expression analysis of mRNA and ncRNA. Genetic regions corresponding to mapped read clusters in exon-intron junctions, introns and intergenes were detected using the NGS Analyzer and defined as ncRNA candidates (Fig. 7c). Moreover, read clusters with different levels between LTED and MCF7 cells, and LTED-RES and LTED cells were extracted using edgeR1.6.15 in the Genomatix Genome Analyzer (Fig. 7d). Genetic regions that were up-regulated in LTED cells compared with that in MCF7 cells were selected on the basis of fold-change ranking (log2 fold-change $<-1.0)$ together with $P$-values $(P<0.01)$, which identified 388 mRNAs and 10,808 ncRNAs (Genomatix Software GmbH). $P$-values were calculated by edgeR algorithm version 1.6 .15 (ref. 68). Similar selections were performed for down-regulated mRNAs and ncRNAs in LTED-RES cells compared with that in LTED cells, resulted in 359 mRNAs and 933 ncRNAs. Overlapping among the four groups was analysed and summarized to show the number of shared genes in a four-way Venn diagram (Genomatix Software GmbH).

Nonspecific enrichments detected in each sample were subtracted from the local enrichments (clusters) representing genomic regions bound in comparative samples.

ChIP-Seq data used in this study. ChIP-Seq data of MCF7 cells were obtained from ENCODE Consortium through UCSC genome browser (University of California, Santa Cruz), and compared with our mRNA-Seq and RNA-Seq data (Figs $4 a$ and $6 a$ and Supplementary Figs $3 a$ and $4 a$ ). The files used in the analyses are listed in Supplementary Table 1.

Fluorescence in situ hybridization. Cells grown on coverslips were fixed with $4 \%$ formaldehyde and $0.5 \%$ Triton X-100 in phosphate-buffered saline (PBS) for $15 \mathrm{~min}$, and then permeabilized with $0.5 \%$ saponin and $0.5 \%$ Triton X-100 in PBS for 20 min. Samples were immersed in $20 \%$ glycerol in PBS for $30 \mathrm{~min}$, and then subjected to four cycles of freeze-thawing by freezing the cells in liquid nitrogen for $30 \mathrm{~s}$ each time. The cells were then treated with $0.1 \mathrm{~N} \mathrm{HCl}$ for $15 \mathrm{~min}$. For denaturation and hybridization, the cells were incubated in hybridization mixtures $\left(2 \times\right.$ SSC, $50 \%$ formamide, $10 \%$ dextran sulfate, $1 \mathrm{mg} \mathrm{ml}^{-1}$ tRNA and $5-10 \mu \mathrm{g} \mathrm{ml}{ }^{-1}$ probe DNA) at $75^{\circ} \mathrm{C}$ for $4-10 \mathrm{~min}$ and then $37^{\circ} \mathrm{C}$ for $48-72 \mathrm{~h}$. $\mathrm{BAC}$ and plasmid probes were labelled with biotin or digoxigenin in a nick translation mixture (Roche) according to the manufacturer's protocol. After hybridization, the cells were washed with $2 \times \mathrm{SSC}$ and $50 \%$ formamide at $37^{\circ} \mathrm{C}$ for $5 \mathrm{~min}$, followed by $2 \times \mathrm{SSC}$ at $37^{\circ} \mathrm{C}$ for $5 \mathrm{~min}$. FISH signals were detected with FITC-anti-digoxigenin (Roche; dilution 1:250) or Cy3-streptavidin (Jackson ImmunoResearch; dilution 1:1,000). For dual-colour FISH, a SPEC ESR1/CEN6 Dual Color Probe kit (ZytoVision) was used. For nuclease treatment, cells were 


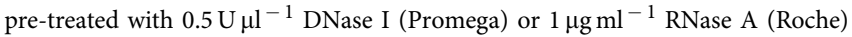
for $30 \mathrm{~min}$ before hybridizations.

For FISH with tissue microarray (BioChain, Z7020005 and Biomax, BR1504), sections were processed using ZytoLight SPEC ESR1/CEN 6 Dual Color Probe Kit (Zytovision, Bremerhaven, Z-2070-20) according to manufacturer's protocol. Briefly, slides were heated for $10 \mathrm{~min}$ at $70^{\circ} \mathrm{C}$, treated with xylene twice for $10 \mathrm{~min}$ at room temperature, rehydrated in a series of graded ethanol solutions, washed with $\mathrm{dH}_{2} \mathrm{O}$, incubated in Pretreatment Solution Citric at $98^{\circ} \mathrm{C}$ for $15 \mathrm{~min}$ and then washed again with $\mathrm{dH}_{2} \mathrm{O}$. The slides were then treated with pepsin solution for $10 \mathrm{~min}$ at $37^{\circ} \mathrm{C}$, and washed with $2 \times \mathrm{SSC}$ solution for $5 \mathrm{~min}$ and with $\mathrm{dH} 2 \mathrm{O}$ for $1 \mathrm{~min}$ at room temperature. The slides were dehydrated, air-dried and incubated with a probe at $76^{\circ} \mathrm{C}$ for $10 \mathrm{~min}$ for denaturation, and at $37^{\circ} \mathrm{C}$ overnight for hybridization. DNA was counterstained with 5,6-diamidino-2-phenylindole (DAPI).

Immunofluorescence and immunohistochemistry. For immunofluorescence, cells were fixed with $4 \%$ paraformaldehyde in PBS for $15 \mathrm{~min}$ at room temperature. The cells were washed three times with PBS for $5 \mathrm{~min}$, then permeabilized with PBS containing $0.2 \%$ Triton X-100 and $0.5 \%$ normal goat serum (GIBCO) for $5 \mathrm{~min}$ on ice. The cells were incubated at room temperature with PBS with $0.5 \%$ normal goat serum (GIBCO) three times for $5 \mathrm{~min}$, then with the primary antibodies for $60 \mathrm{~min}$, followed by the Cy3- or Alexa488-conjugated secondary antibodies for $60 \mathrm{~min}$. The cells were washed with PBS three times for $10 \mathrm{~min}$ each. DNA was counterstained with $1 \mu \mathrm{g} \mathrm{ml}^{-1}$ DAPI.

For immunohistochemical staining, sections in a tissue microarray (Biomax, BR1504) were processed with automated IHC staining system, BenchMark XT (Ventana). The sections were de-paraffinized in EZ prep (Ventana) at $72{ }^{\circ} \mathrm{C}$ for 4 min and then incubated in Immunoblock (DS Pharma Biomedicals) at $37^{\circ} \mathrm{C}$ for $12 \mathrm{~min}$ to block endogenous peroxidase. Antigens were retrieved by incubating $95^{\circ} \mathrm{C}$ for $8 \mathrm{~min}$, then treated with rabbit monoclonal anti-human ER $\alpha$ clone SP1 (Ventana, $790-4325$, used without dilution) at $37^{\circ} \mathrm{C}$ for $36 \mathrm{~min}$. The antigens were visualized by avidin-biotin-peroxidase complex method using iVIEW DAB detection kit (Ventana). The slides were then counterstained with haematoxylin at $37^{\circ} \mathrm{C}$ for $8 \mathrm{~min}$.

Microscopy and image analysis. Images were obtained with an IX-71 microscope (Olympus) equipped with a $\times 60$ NA1.0 Plan Apo objective lens, a cooled CCD camera (Hamamatsu) and image acquisition software (Lumina Vision Version 2.4; Mitani Corporation). For imaging cytometry analyses of FISH, image stacks of three-dimensional data sets were collected at $0.5-1.0 \mu \mathrm{m}$ intervals through the $z$ axis, subjected to projections and used for automatic detection and counting of FISH signals with CELAVIEW RS100 software (Olympus) and Cellomics CellInsight (Thermo Fisher Scientific). Images in Figs $1 \mathrm{c}$ and $2 \mathrm{c}$ and Supplementary Fig. 1c were obtained with a confocal laser-scanning microscope (LSM 780, Carl Zeiss) equipped with $\times 63 / 1.4$ Plan-Apochrome objective lens and a cooled CCD camera (Carl Zeiss). Image acquisition was done using LSM software (Carl Zeiss).

Immunoblotting. To prepare total cell lysate, cells were dissolved in SDS sample buffer containing benzonase (Sigma). Proteins were separated by SDSpolyacrylamide gel electrophoresis and then transferred to a nitrocellulose membrane, Amersham Hybond ECL (GE Healthcare). The membrane was blocked for $1 \mathrm{~h}$ with PBS containing $10 \%$ nonfat dry milk and then incubated with primary antibodies in PBS containing $0.03 \%$ Tween 20 for $1 \mathrm{~h}$. The membrane was washed with PBS containing $0.3 \%$ Tween 20 three times for $10 \mathrm{~min}$ each, and incubated with horseradish peroxidase-conjugated secondary antibodies for $40 \mathrm{~min}$. After the membrane was washed with PBS containing $0.3 \%$ Tween 20 three times for $10 \mathrm{~min}$ each, signals were visualized with Western Lightning Plus-ECL (PerkinElmer). Uncropped immunoblot images for Figs 4e and 5b, and Supplementary Fig. 6a are provided in Supplementary Figs 8 and 9, respectively.

PCR with reverse transcription. Total RNA was isolated from cultured cells with TRIzol (Invitrogen) and then treated with DNase I (Roche) before cDNA synthesis. Reverse transcription was carried out with a High Capacity cDNA reverse transcription kit (Applied Biosystems). qRT-PCR was performed with SYBR green fluorescence on an ABI Prism 7300 system (Applied Biosystems). Values were normalized to $\beta$-actin or glyceraldehyde-3-phosphate dehydrogenase (GAPDH) gene expression before calculating relative fold changes. The amplification efficiency for each primer set was measured using genomic DNA and used for normalization in Figs $2 \mathrm{a}$ and $4 \mathrm{~b}$. To indicate absolute expression levels of each RNA in Figs $2 \mathrm{a}$,e and $4 \mathrm{~b}, \Delta \mathrm{C}_{\mathrm{t}}$ values are summarized in Supplementary Table 6. Primer sequences are listed in Supplementary Table 3. For RT-PCR, amplified products were separated by electrophoresis on $2 \%$ agarose gels and stained with ethidium bromide. All PCR amplifications were performed within a quantitative range by adjusting the cycle numbers. For RT-PCR in Supplementary Fig. 3b, PCR-amplified products were run on agarose gel and stained with ethidium bromide. Corresponding uncropped gel images are provided in Supplementary Fig. 9.
Chromatin immunoprecipitation-qPCR. MCF7 and LTED cells were crosslinked with $1 \%$ formaldehyde at $37^{\circ} \mathrm{C}$ for $10 \mathrm{~min}$. Crude cell lysates were sonicated to generate DNA fragments of 200-500 bp using a Bioruptor USD-250 (Cosmo-Bio; 15 sonications of $30 \mathrm{~s}$ with $30 \mathrm{~s}$ intervals at $250 \mathrm{~W}$ ). Chromatin was precipitated with antibodies at $4{ }^{\circ} \mathrm{C}$ overnight, washed and de-crosslinked for $5 \mathrm{~h}$. DNA enrichment in ChIP samples was determined by qPCR with SYBR green fluorescence on an ABI Prism 7300 system. The threshold was set to cross a point where PCR amplification was linear, and the cycle number required to reach the threshold was recorded and analysed using Microsoft Excel. PCR was performed using precipitated DNA and the input DNA. Primers used in ChIP-qPCR are listed in Supplementary Table 3.

RNA interference. Cells were transfected with specific siRNAs (Nippon EGT) using RNAiMAX (Invitrogen). Target sequences for each siRNA are listed in Supplementary Table 3 . The cells were analysed at 48 and $72 \mathrm{~h}$ after transfection.

Cell counting. The number of proliferating cells was counted using an automatic cell imaging counter (CYTORECON; GE Healthcare) or image cytometer (Cellomics CellInsight; Thermo Fisher Scientific).

3C assay. Formaldehyde-crosslinked chromatin from MCF7 and LTED cells was digested with $B g l I I$ restriction enzyme overnight, followed by ligation with T4 DNA ligase at $16^{\circ} \mathrm{C}$ for $4 \mathrm{~h}$. To prepare control templates for standard curves, a BAC clone covering the ESR1 locus (RP11-450E24 and RP3-404G5) was digested with BglII, followed by random religation. After reversing the cross-links, genomic DNA was purified by phenol extraction and ethanol precipitation. The ligated products were assessed by qPCR with the ABI Prism 7300 and Thunderbird SYBR qPCR Mix (Toyobo). The efficiency of BglII digestion was evaluated by qPCR using primers that only amplified undigested DNA fragments containing the BglII site. More than $80 \%$ of the individual restriction sites were digested under the experimental condition. 3C-qPCR data were normalized to a loading control that was obtained with primers that amplified a genomic fragment in the ESR1 locus (intron 3 ), which represented the amount of template DNA. Primers used in the 3C assay are listed in Supplementary Table 3.

Overexpression of SIRT1 in LTED cells. Full-length cDNA for human SIRT1 was amplified by PCR using primers described in Supplementary Table 3. The amplified fragments were digested with EcoRV and $\mathrm{XbaI}$, and then cloned into pcDNA3-myc (pcDNA3-myc-SIRT1). LTED cells $\left(1 \times 10^{5}\right.$ cells) were transfected with the plasmid $(2 \mu \mathrm{g})$ using $5 \mu \mathrm{l} \mathrm{FuGene} \mathrm{HD}$ (Roche Applied Science) in a sixwell plate for $48 \mathrm{~h}$, and then subjected to immunofluorescence or immuno-FISH.

Statistical analysis. Comparisons between groups were analysed using the two-tailed Student's $t$-test. A value of $P<0.05$ was considered statistically significant. For RNA- and mRNA-Seq experiments, $P$-values were calculated with DESeq and edge $\mathrm{R}^{67,68}$.

\section{References}

1. Sotiriou, C. \& Piccart, M. J. Taking gene-expression profiling to the clinic: when will molecular signatures become relevant to patient care? Nat. Rev. Cancer 7, 545-553 (2007).

2. Ali, S. \& Coombes, R. C. Endocrine-responsive breast cancer and strategies for combating resistance. Nat. Rev. Cancer 2, 101-112 (2002).

3. Yager, J. D. \& Davidson, N. E. Estrogen carcinogenesis in breast cancer. N. Engl. J. Med. 354, 270-282 (2006)

4. Musgrove, E. A. \& Sutherland, R. L. Biological determinants of endocrine resistance in breast cancer. Nat. Rev. Cancer 9, 631-643 (2009).

5. Katzenellenbogen, B. S., Kendra, K. L., Norman, M. J. \& Berthois, Y. Proliferation, hormonal responsiveness, and estrogen receptor content of MCF-7 human breast cancer cells grown in the short-term and long-term absence of estrogens. Cancer Res. 47, 4355-4360 (1987).

6. Welshons, W. V. \& Jordan, V. C. Adaptation of estrogen-dependent MCF-7 cells to low estrogen (phenol red-free) culture. Eur. J. Cancer Clin. Oncol. 23, 1935-1939 (1987).

7. Santen, R. J. et al. Long-term estradiol deprivation in breast cancer cells up-regulates growth factor signaling and enhances estrogen sensitivity. Endocr. Relat. Cancer 12(Suppl 1): S61-S73 (2005).

8. Jeng, M. H. et al. Estrogen receptor expression and function in long-term estrogen-deprived human breast cancer cells. Endocrinology 139, 4164-4174 (1998).

9. Chan, C. M., Martin, L. A., Johnston, S. R., Ali, S. \& Dowsett, M. Molecular changes associated with the acquisition of oestrogen hypersensitivity in MCF-7 breast cancer cells on long-term oestrogen deprivation. J. Steroid Biochem. Mol. Biol. 81, 333-341 (2002). 
10. Martin, L. A. et al. Enhanced estrogen receptor (ER) alpha, ERBB2, and MAPK signal transduction pathways operate during the adaptation of MCF-7 cells to long term estrogen deprivation. J. Biol. Chem. 278, 30458-30468 (2003).

11. Martin, L. A., Farmer, I., Johnston, S. R., Ali, S. \& Dowsett, M. Elevated ERK1/ERK2/estrogen receptor cross-talk enhances estrogen-mediated signaling during long-term estrogen deprivation. Endocr. Relat. Cancer 12 (Suppl 1): S75-S84 (2005).

12. Ellis, M. J. et al. Lower-dose vs high-dose oral estradiol therapy of hormone receptor-positive, aromatase inhibitor-resistant advanced breast cancer: a phase 2 randomized study. J. Am. Med. Assoc. 302, 774-780 (2009).

13. Lonning, P. E. et al. High-dose estrogen treatment in postmenopausal breast cancer patients heavily exposed to endocrine therapy. Breast Cancer Res. Treat. 67, 111-116 (2001)

14. Iwase, H. et al. Ethinylestradiol is beneficial for postmenopausal patients with heavily pre-treated metastatic breast cancer after prior aromatase inhibitor treatment: a prospective study. Br. J. Cancer 109, 1537-1542 (2013).

15. Okamoto, I., Otte, A. P., Allis, C. D., Reinberg, D. \& Heard, E. Epigenetic dynamics of imprinted $\mathrm{X}$ inactivation during early mouse development. Science 303, 644-649 (2004).

16. Hebbes, T. R., Clayton, A. L., Thorne, A. W. \& Crane-Robinson, C. Core histone hyperacetylation co-maps with generalized DNase I sensitivity in the chicken beta-globin chromosomal domain. EMBO J. 13, 1823-1830 (1994).

17. Saitoh, N. et al. Structural and functional conservation at the boundaries of the chicken beta-globin domain. EMBO J. 19, 2315-2322 (2000).

18. Stalder, J. et al. Tissue-specific DNA cleavages in the globin chromatin domain introduced by DNAase I. Cell 20, 451-460 (1980).

19. Dixon, J. R. et al. Topological domains in mammalian genomes identified by analysis of chromatin interactions. Nature 485, 376-380 (2012).

20. Nora, E. P. et al. Spatial partitioning of the regulatory landscape of the X-inactivation centre. Nature 485, 381-385 (2012).

21. Nora, E. P., Dekker, J. \& Heard, E. Segmental folding of chromosomes: a basis for structural and regulatory chromosomal neighborhoods? Bioessays 35, 818-828 (2013).

22. Bickmore, W. A. \& van Steensel, B. Genome architecture: domain organization of interphase chromosomes. Cell 152, 1270-1284 (2013).

23. Geisler, S. \& Coller, J. RNA in unexpected places: long non-coding RNA functions in diverse cellular contexts. Nat. Rev. Mol. Cell Biol. 14, 699-712 (2013).

24. Carninci, P. et al. The transcriptional landscape of the mammalian genome. Science 309, 1559-1563 (2005).

25. Ravasi, T. et al. Experimental validation of the regulated expression of large numbers of non-coding RNAs from the mouse genome. Genome Res. 16, 11-19 (2006).

26. Djebali, S. et al. Landscape of transcription in human cells. Nature 489, 101-108 (2012)

27. Lee, J. T. Epigenetic regulation by long noncoding RNAs. Science 338, 1435-1439 (2012)

28. Gehm, B. D., McAndrews, J. M., Chien, P. Y. \& Jameson, J. L. Resveratrol, a polyphenolic compound found in grapes and wine, is an agonist for the estrogen receptor. Proc. Natl Acad. Sci. USA 94, 14138-14143 (1997).

29. Nwachukwu, J. C. et al. Resveratrol modulates the inflammatory response via an estrogen receptor-signal integration network. eLife 3, e02057 (2014).

30. Yoon, D. S. et al. Variable levels of chromosomal instability and mitotic spindle checkpoint defects in breast cancer. Am. J. Pathol. 161, 391-397 (2002).

31. Aguilar, H. et al. Biological reprogramming in acquired resistance to endocrine therapy of breast cancer. Oncogene 29, 6071-6083 (2010).

32. Miller, T. W. et al. Hyperactivation of phosphatidylinositol-3 kinase promotes escape from hormone dependence in estrogen receptor-positive human breast cancer. J. Clin. Invest. 120, 2406-2413 (2010).

33. Perou, C. M. et al. Molecular portraits of human breast tumours. Nature 406, 747-752 (2000).

34. Sorlie, T. et al. Repeated observation of breast tumor subtypes in independent gene expression data sets. Proc. Natl Acad. Sci. USA 100, 8418-8423 (2003).

35. Kos, M., Reid, G., Denger, S. \& Gannon, F. Minireview: genomic organization of the human ERalpha gene promoter region. Mol. Endocrinol. 15, 2057-2063 (2001).

36. Reid, G., Denger, S., Kos, M. \& Gannon, F. Human estrogen receptor-alpha: regulation by synthesis, modification and degradation. Cell. Mol. Life. Sci. 59, 821-831 (2002)

37. Kim, T. K. et al. Widespread transcription at neuronal activity-regulated enhancers. Nature 465, 182-187 (2010).

38. Harrow, J. et al. GENCODE: the reference human genome annotation for The ENCODE Project. Genome Res. 22, 1760-1774 (2012).

39. Kent, W. J. et al. The human genome browser at UCSC. Genome Res. 12, 996-1006 (2002).

40. Heintzman, N. D. et al. Distinct and predictive chromatin signatures of transcriptional promoters and enhancers in the human genome. Nat. Genet. 39, 311-318 (2007)
41. rMay, D. et al. Large-scale discovery of enhancers from human heart tissue. Nat. Genet. 44, 89-93 (2012).

42. Ellison-Zelski, S. J., Solodin, N. M. \& Alarid, E. T. Repression of ESR1 through actions of estrogen receptor alpha and Sin3A at the proximal promoter. Mol. Cell. Biol. 29, 4949-4958 (2009).

43. Hu, X. F. et al. Circumvention of tamoxifen resistance by the pure anti-estrogen ICI 182,780. Int. J. Cancer 55, 873-876 (1993).

44. Dunbier, A. K. et al. ESR1 is co-expressed with closely adjacent uncharacterised genes spanning a breast cancer susceptibility locus at 6q25.1. PLoS Genet. 7, e1001382 (2011).

45. Solier, S. et al. Transcription poisoning by Topoisomerase I is controlled by gene length, splice sites, and miR-142-3p. Cancer Res. 73, 4830-4839 (2013).

46. Eisenberg, E. \& Levanon, E. Y. Human housekeeping genes are compact. Trends Genet. 19, 362-365 (2003).

47. King, I. F. et al. Topoisomerases facilitate transcription of long genes linked to autism. Nature 501, 58-62 (2013).

48. Castles, C. G., Oesterreich, S., Hansen, R. \& Fuqua, S. A. Auto-regulation of the estrogen receptor promoter. J. Steroid Biochem. Mol. Biol. 62, 155-163 (1997)

49. Jia, H. et al. Genome-wide computational identification and manual annotation of human long noncoding RNA genes. RNA 16, 1478-1487 (2010).

50. Ashe, H. L., Monks, J., Wijgerde, M., Fraser, P. \& Proudfoot, N. J. Intergenic transcription and transinduction of the human beta-globin locus. Genes Dev. 11, 2494-2509 (1997).

51. Vallot, C. et al. XACT, a long noncoding transcript coating the active X chromosome in human pluripotent cells. Nat. Genet. 45, 239-241 (2013).

52. Nagano, T. et al. The Air noncoding RNA epigenetically silences transcription by targeting G9a to chromatin. Science 322, 1717-1720 (2008).

53. Schubert, T. et al. Df31 protein and snoRNAs maintain accessible higher-order structures of chromatin. Mol. Cell 48, 434-444 (2012).

54. Hall, L. L. et al. Stable C0T-1 repeat RNA is abundant and is associated with euchromatic interphase chromosomes. Cell 156, 907-919 (2014).

55. Marahrens, Y., Panning, B., Dausman, J., Strauss, W. \& Jaenisch, R. Xistdeficient mice are defective in dosage compensation but not spermatogenesis. Genes Dev. 11, 156-166 (1997)

56. Kopczynski, C. C. \& Muskavitch, M. A. Introns excised from the Delta primary transcript are localized near sites of Delta transcription. J. Cell Biol. 119, 503-512 (1992).

57. Guil, S. et al. Intronic RNAs mediate EZH2 regulation of epigenetic targets Nat. Struct. Mol. Biol. 19, 664-670 (2012).

58. Gardner, E. J., Nizami, Z. F., Talbot, Jr C. C. \& Gall, J. G. Stable intronic sequence RNA (sisRNA), a new class of noncoding RNA from the oocyte nucleus of Xenopus tropicalis. Genes Dev. 26, 2550-2559 (2012).

59. Jensen, T. H., Jacquier, A. \& Libri, D. Dealing with pervasive transcription. Mol. Cell 52, 473-484 (2013).

60. Holst, F. et al. Estrogen receptor alpha (ESR1) gene amplification is frequent in breast cancer. Nat. Genet. 39, 655-660 (2007).

61. Tomita, S. et al. Estrogen receptor alpha gene ESR1 amplification may predict endocrine therapy responsiveness in breast cancer patients. Cancer Sci. 100, 1012-1017 (2009).

62. Ooi, A. et al. Gene amplification of ESR1 in breast cancers--fact or fiction? A fluorescence in situ hybridization and multiplex ligation-dependent probe amplification study. J. Pathol. 227, 8-16 (2012).

63. Magnani, L. et al. Genome-wide reprogramming of the chromatin landscape underlies endocrine therapy resistance in breast cancer. Proc. Natl Acad. Sci. USA 110, E1490-E1499 (2013).

64. Yaswen, P. \& Stampfer, M. R. Molecular changes accompanying senescence and immortalization of cultured human mammary epithelial cells. Int. J. Biochem. Cell. Biol. 34, 1382-1394 (2002).

65. Trapnell, C., Pachter, L. \& Salzberg, S. L. TopHat: discovering splice junctions with RNA-Seq. Bioinformatics 25, 1105-1111 (2009).

66. Trapnell, C. et al. Transcript assembly and quantification by RNA-Seq reveals unannotated transcripts and isoform switching during cell differentiation. Nat. Biotechnol. 28, 511-515 (2010).

67. Anders, S. \& Huber, W. Differential expression analysis for sequence count data. Genome Biol. 11, R106 (2010).

68. Robinson, M. D. \& Smyth, G. K. Small-sample estimation of negative binomia dispersion, with applications to SAGE data. Biostatistics 9, 321-332 (2008).

\section{Acknowledgements}

We thank all the members of our laboratory for discussions and technical assistance, Takumi Takizawa (Gunma University), Hideyuki Tanabe (The Graduate University for Advanced Studies), Sui Huang (Northwestern University), Christian Lanctot (Charles University in Prague), Gary Felsenfeld, Takashi Ideue (Kumamoto University) and Masahiko Harata (Tohoku University) for valuable advice, and Sakura Aoki (CTC Laboratory Systems) and Takehiko Oka (World Fusion) for helping with the bioinformatic analyses of the sequencing data. This work was supported by grants from the Ministry of Education, Culture, Sports, Science and Technology of Japan and the Japan Science and Technology Agency (CREST; M.N. and N.S.). 


\section{Author contributions}

S.T., M.O.A.A., N.S. and M.N. designed and conducted the experiments, and prepared the manuscripts, together with support from S.F., H.M. and H.I. K.M. and Y.O. conducted and analysed high-throughput sequencing experiments.

\section{Additional information}

Accession codes: mRNA-Seq and RNA-Seq data sets have been deposited in the DNA Data Bank of Japan (DDBJ) Sequence Read Archive (accession number: DRA001006).

Supplementary Information accompanies this paper at http://www.nature.com/ naturecommunications

Competing financial interests: The authors declare no competing financial interests.
Reprints and permission information is available online at http://npg.nature.com/ reprintsandpermissions/

How to cite this article: Tomita, S. et al. A cluster of noncoding RNAs activates the ESR 1 locus during breast cancer adaptation. Nat. Commun. 6:6966 doi: 10.1038/ncomms7966 (2015).

(c) (i) This work is licensed under a Creative Commons Attribution 4.0 International License. The images or other third party material in this article are included in the article's Creative Commons license, unless indicated otherwise in the credit line; if the material is not included under the Creative Commons license, users will need to obtain permission from the license holder to reproduce the material. To view a copy of this license, visit http://creativecommons.org/licenses/by/4.0/ 